\title{
Scalable Algorithms for Large Competing Risks Data
}

\author{
Eric S. Kawaguchi ${ }^{1}$ \\ Jenny I. Shen ${ }^{2}$ \\ Marc A. Suchard ${ }^{3,4,5}$ \\ and Gang $\mathrm{Li}^{3,4, *}$ \\ ${ }^{1}$ Division of Biostatistics and Epidemiology, University of Southern California \\ 2 Division of Nephrology and Hypertension \\ Los Angeles Biomedical Institute at Harbor-UCLA Medical Center \\ ${ }^{3}$ Department of Biostatistics, University of California, Los Angeles \\ ${ }^{4}$ Department of Biomathematics, University of California, Los Angeles \\ ${ }^{5}$ Department of Human Genetics, University of California Los Angeles \\ *email: vli@ucla.edu
}

\begin{abstract}
This paper develops two orthogonal contributions to scalable sparse regression for competing risks time-to-event data. First, we study and accelerate the broken adaptive ridge method (BAR), an $\ell_{0}$-based iteratively reweighted $\ell_{2}$-penalization algorithm that achieves sparsity in its limit, in the context of the Fine-Gray (1999) proportional subdistributional hazards (PSH) model. In particular, we derive a new algorithm for BAR regression, named cycBAR, that performs cyclic update of each coordinate using an explicit thresholding formula. The new cycBAR algorithm effectively avoids fitting multiple reweighted $\ell_{2}$-penalizations and thus yields impressive speedups over the original BAR algorithm. Second, we address a pivotal computational issue related to fitting the PSH model. Specifically, the computation costs of the log-pseudo likelihood and its derivatives for PSH model grow at the rate of $O\left(n^{2}\right)$ with the sample size $n$ in current implementations. We propose a novel forwardbackward scan algorithm that reduces the computation costs to $O(n)$. The proposed
\end{abstract}


method applies to both unpenalized and penalized estimation for the PSH model and has exhibited drastic speedups over current implementations. Finally, combining the two algorithms can yields $>1,000$ fold speedups over the original BAR algorithm. Illustrations of the impressive scalability of our proposed algorithm for large competing risks data are given using both simulations and a United States Renal Data System data.

Keywords: Broken Adaptive Ridge; Fine-Gray model; $\ell_{0}$-regularization; Massive Sample Size; Model Selection/Variable selection; Oracle property; Subdistribution hazard. 


\section{Introduction}

Advancing informatics tools make large-scale data such as electronic health record (EHR) data and genomic data routinely accessible to researchers. This data deluge offers unprecedented opportunities for new and innovative approaches to improve research and learning (Schuemie et al., 2017). However, it also presents new computational challenges and barriers for quantitative researchers as many current statistical methodologies and computational tools may grind to a halt as the sample size $(n)$ and/or number of covariates $\left(p_{n}\right)$ grow

large. Such challenges are particularly common in time-to-event data analysis where the likelihood function (such as the partial likelihood for the Cox model with data) and its derivatives typically require $O\left(n^{2}\right)$ number of operations, which will explode quickly as $n$ increases. The computational burden can be further aggravated as the number of covariates $\left(p_{n}\right)$ increases. Statistical methods coupled with high-performance algorithms are critically needed for large-scale time-to-event data analysis.

This paper aims to develop high-performance computational methods for large-scale competing risks time-to-event data analysis by addressing two orthogonal computational challenges due to large $p_{n}$ and large $n$ respectively. First, we develop a scalable $\ell_{0}$-based method for simultaneous variable selection and parameter estimation for the large $p_{n}$ problem. It is well known that $\ell_{0}$-penalized regression is natural for variable selection, but is computationally NP hard and not scalable to even moderate $p_{n}$. As a scalable approximation to $\ell_{0}$-penalized regression, the broken adaptive ridge $\mathrm{BAR}$ estimator, defined as the limit of an $\ell_{0}$-based iteratively reweighted $\ell_{2}$-penalization algorithm, has been recently studied for simultaneous variable selection and parameter estimation and shown to possess some desirable selection, estimation, and grouping properties under various model settings (see, e.g., Zhao et al. (2018), Dai et al. (2018), Zhao et al. (2019), and Zhao et al. (2019)). However, while previous research has focused on studying the statistical properties of BAR 
methodology, the feasibility of applying BAR to large-scale data has yet to be explored. To this end, we note that BAR requires fitting multiple reweighted $\ell_{2}$-penalized regressions until convergence, which can potentially create a computational bottleneck if a large number of iterations is needed for convergence. As demonstrated in Table 1 of Section 4. BAR can grind to a halt for large scale data. Second, we address a pivotal computational issue specifically related to fitting the PSH model when $n$ is large. As discussed later in Section 2.4, computation of the log-pseudo likelihood and its derivatives for the PSH model involves $O\left(n^{2}\right)$ number of operations, which presents a critical computational barrier as $n$ becomes large. Moreover, because the computations involve weighted sums over some risk sets where the risks sets are not monotone over time and the weights are subject-specific, commonly used efficient computational techniques for fitting the Cox (1972) model do not apply to the PSH model. To the best of our knowledge, no algorithm exists in the literature that reduces the computational cost for the PSH model from $O\left(n^{2}\right)$ to a lower order.

In addressing the aforementioned computational challenges for large data, the contribution of this paper is two folds:

1. We propose a novel cyclic coordinate-wise update algorithm for BAR, referred to as CYCBAR, by deriving an explicit analytic coordinate-wise update for a fixed-point problem whose unique solution approximates the BAR estimator. Because the CYCBAR algorithm avoids carrying out iteratively reweighted $\ell_{2}$-penalizations, it can result in substantial gains in computational efficiency. We emphasize that the application of the CYCBAR algorithm over the original BAR method is not limited to the PSH model and spans a variety of models and data settings such as generalized linear models and time-to-event models, as well as sparse signal reconstruction Gorodnitsky and Rao, 1997) and compressive sensing (Candes et al., 2008; Chartrand and Yin, 2008; Gasso et al., 2009: Daubechies et al., 2010; Wipf and Nagarajan, 2010) where 
the $\ell_{0}$-based iteratively reweighted $\ell_{2}$-penalization algorithm are popularly used. In our numerical studies (Section 3.3, Figure 1(b)), CYCBAR showed marked reduction in runtime over the standard BAR.

2. By exploiting the special structure of the risk set and the subject-specific weight functions associated with the Fine-Gray pseudo likelihood and its derivatives, we derive a novel forward-backward scan algorithm to reduce their computational costs from $O\left(n^{2}\right)$ to $O(n)$, allowing one to analyze competing risks data much quicker than current approaches. We have observed in empirical studies, e.g. Figure 1(c) in Section 3.3, that the forward-backward scan algorithm can yield dramatic speedups over standard implementations. We point out that our proposed forward-backward scan algorithm for the PSH model is not specific to the BAR method and can be applied to accelerate other penalized regression methods such as LASSO (Tibshirani, 1996), SCAD (Fan and Li, 2001), adaptive LASSO (Zou, 2006), and MCP (Zhang, 2010) for the PSH model (Fu et al., 2017), and the unpenalized estimation method of Fine and Gray (1999), as well as to hypothesis testing and cumulative incidence estimation for the PSH model.

The rest of this article is organized as follows. In Section 2.1, we review the mathematical formulation of competing risks data and the Fine and Gray (1999) proportional subdistribution hazards model. Section 2.2, introduces the BAR estimator for the PSH model and refers its asymptotic properties to the Online Supplementary Material. Section 2.3 derives the cyclic coordinate-wise BAR algorithm. The forward-backward scan method for the PSH model is described in Section 2.4. Section 3 presents some simulation studies to demonstrate the computational efficiency gains of both the CYCBAR and forward-backward scan algorithms. A proof-of-concept real data example for fitting largescale competing risks data is provided in Section 4 using a subset of the United States 
Renal Data System (USRDS). Concluding remarks are given in Section 5. The proposed method has been implemented in an $\mathrm{R}$ package, named pshBAR, which is available at https://github.com/erickawaguchi/pshBAR.

\section{Methodology}

\subsection{Competing risks data, model, and parameter estimation}

Competing risks time-to-event data arises frequently in clinical trials, reliability testing, social science, and many other fields (Prentice et al., 1978; Pintilie, 2006; Putter et al., 2007). Competing risks occur when individuals are susceptible to more than one types of possibly correlated events or causes and the occurrence of one event precludes the others from happening. For example, one may wish to study time until first kidney transplant for kidney dialysis patients with end-stage renal disease. Then terminating events such as death, renal function recovery, or discontinuation of dialysis are competing risks as their occurrence will prevent subjects from receiving a transplant. For $i=1, \ldots, n$, let $T_{i}, C_{i}$, $\epsilon_{i}$, and $\mathbf{z}_{i}$ be the event time, possible right-censoring time, cause (event type), and a $p_{n^{-}}$ dimensional vector of time-independent covariates, respectively, for subject $i$. Without loss of generality assume there are two event types $\epsilon \in\{1,2\}$ where $\epsilon=1$ is the event of interest and $\epsilon=2$ is the competing risk. With the presence of right censoring, we generally observe $X_{i}=T_{i} \wedge C_{i}, \delta_{i}=I\left(T_{i} \leq C_{i}\right)$, where $a \wedge b=\min (a, b)$ and $I(\cdot)$ is the indicator function. Competing risks data consists of $n$ independent and identically distributed quadruplets $\left\{\left(X_{i}, \delta_{i}, \delta_{i} \epsilon_{i}, \mathbf{z}_{i}\right\}_{i=1}^{n}\right.$. Assume that there exists a $\tau$ such that (1) for some arbitrary time $t$, $t \in[0, \tau] ;(2) \operatorname{Pr}\left(T_{i}>\tau\right)>0$ and $\operatorname{Pr}\left(C_{i}>\tau\right)>0$ for all $i=1, \ldots, n$.

An important quantity for competing risks data is the cumulative incidence function (CIF), which describes the probability of failing from a certain cause of interest before 
the other causes. The CIF for cause 1 events conditional on the covariates is defined as $F_{1}(t ; \mathbf{z})=\operatorname{Pr}(T \leq t, \epsilon=1 \mid \mathbf{z})$. To model $F_{1}(t ; \mathbf{z})$, Fine and Gray (1999) introduced the now popular proportional subdistribution hazards (PSH) model:

$$
h_{1}(t \mid \mathbf{z})=h_{10}(t) \exp \left(\mathbf{z}^{\prime} \boldsymbol{\beta}\right)
$$

where

$h_{1}(t \mid \mathbf{z})=\lim _{\Delta t \rightarrow 0} \frac{\operatorname{Pr}\{t \leq T \leq t+\Delta t, \epsilon=1 \mid T \geq t \cup(T \leq t \cap \epsilon \neq 1), \mathbf{z}\}}{\Delta t}=-\frac{d}{d t} \log \left\{1-F_{1}(t ; \mathbf{z})\right\}$

is a subdistribution hazard (Gray, 1988), $h_{10}(t)$ is a completely unspecified baseline subdistribution hazard, and $\boldsymbol{\beta}$ is a $p_{n} \times 1$ vector of regression coefficients. As Fine and Gray (1999) mentioned, the risk set associated with $h_{1}(t ; \mathbf{z})$ is somewhat counterfactual as it includes subjects who are still at risk $(T \geq t)$ and those who have already observed the competing risk prior to time $t(T \leq t \cap \epsilon \neq 1)$. However, this construction is useful for direct modeling of the CIF.

Inference for the PSH model based on the following log-pseudo likelihood (Fine and Gray, 1999):

$$
l(\boldsymbol{\beta})=\sum_{i=1}^{n} \int_{0}^{\tau}\left(\mathbf{z}_{i}^{\prime} \boldsymbol{\beta}-\log \left\{\sum_{j} \hat{w}_{j}(s) Y_{j}(s) \exp \left(\mathbf{z}_{j}^{\prime} \boldsymbol{\beta}\right)\right\}\right) \times \hat{w}_{i}(s) d N_{i}(s),
$$

where $N_{i}(t)=I\left(T_{i} \leq t, \epsilon_{i}=1\right), Y_{i}(t)=1-N_{i}(t-), \hat{w}_{i}(t)$ is a time-dependent weight for subject $i$ at time $t$ defined as $\hat{w}_{i}(t)=I\left(C_{i} \geq T_{i} \wedge t\right) \hat{G}(t) / \hat{G}\left(X_{i} \wedge t\right)$, and $\hat{G}(t)$ is the Kaplan and Meier (1958) estimate for $G(t)=\operatorname{Pr}(C \geq t)$, the survival function of the censoring variable $C$. Note that, for any subject $i$ and time $t, \hat{w}_{i}(t) Y_{i}(t)=0$ if an individual is right censored or has experienced the event of interest; and $\hat{w}_{i}(t) Y_{i}(t)=1$ if $t<X_{i}$, and $\hat{w}_{i}(t) Y_{i}(t)=\hat{G}(t) / \hat{G}\left(X_{i}\right)$ for events due to the competing risk. 
Commonly-used optimization routines to estimate the parameters of the PSH model typically require the calculation of the log-pseudo likelihood (2), the score function

$$
i_{j}(\boldsymbol{\beta})=\sum_{i=1}^{n} I\left(\delta_{i} \epsilon_{i}=1\right) z_{i j}-\sum_{i=1}^{n} I\left(\delta_{i} \epsilon_{i}=1\right) \frac{\sum_{k \in R_{i}} z_{k j} \tilde{w}_{i k} \exp \left(\eta_{k}\right)}{\sum_{k \in R_{i}} \tilde{w}_{i k} \exp \left(\eta_{k}\right)},
$$

and, in some cases, the Hessian diagonals

$$
\ddot{l}_{j j}(\boldsymbol{\beta})=\sum_{i=1}^{n} I\left(\delta_{i} \epsilon_{i}=1\right)\left[\frac{\sum_{k \in R_{i}} z_{k j}^{2} \tilde{w}_{i k} \exp \left(\eta_{k}\right)}{\sum_{k \in R_{i}} \tilde{w}_{i k} \exp \left(\eta_{k}\right)}-\left\{\frac{\sum_{k \in R_{i}} z_{k j} \tilde{w}_{i k} \exp \left(\eta_{k}\right)}{\sum_{k \in R_{i}} \tilde{w}_{i k} \exp \left(\eta_{k}\right)}\right\}^{2}\right],
$$

where

$$
\tilde{w}_{i k}=\hat{w}_{k}\left(X_{i}\right)=\hat{G}\left(X_{i}\right) / \hat{G}\left(X_{i} \wedge X_{k}\right), \quad k \in R_{i},
$$

$R_{i}=\left\{y:\left(X_{y} \geq X_{i}\right) \cup\left(X_{y} \leq X_{i} \cap \epsilon_{y}=2\right)\right\}$ and $\eta_{k}=\mathbf{z}_{k}^{\prime} \boldsymbol{\beta}$. Direct calculations using

the above formulas will need $O\left(n^{2}\right)$ operations due to the the double summations and is computationally taxing for large $n$. We will show how to calculate the double summation linearly in Section 2.4, allowing us to calculate these quantities in $O(n)$ time.

\subsection{Broken adaptive ridge estimation for the proportional sub- distribution hazards model}

Penalized regression is useful for simultaneous variable selection and parameter estimation and has recently been introduced to the PSH model for competing risks data (Ha et al. 2014; Fu et al., 2017; Ahn et al., 2018; Hou et al., 2018). Below we extend the broken adaptive ridge (BAR) estimator to the PSH model.

Let $l(\boldsymbol{\beta})$ be the log-pseudo likelihood defined by (2). The BAR estimator of $\boldsymbol{\beta}$ starts with an initial $\ell_{2}$-penalized (or ridge) estimator

$$
\hat{\boldsymbol{\beta}}^{(0)}=\arg \min _{\boldsymbol{\beta}}\left\{-2 l(\boldsymbol{\beta})+\xi_{n} \sum_{j=1}^{p} \beta_{j}^{2}\right\}
$$


which is updated iteratively by a reweighted $\ell_{2}$-penalized estimator

$$
\hat{\boldsymbol{\beta}}^{(k)}=\arg \min _{\boldsymbol{\beta}}\left\{-2 l(\boldsymbol{\beta})+\lambda_{n} \sum_{j=1}^{p} \frac{\beta_{j}^{2}}{\left|\hat{\beta}_{j}^{(k-1)}\right|^{2}}\right\}, \quad k \geq 1,
$$

where $\xi_{n}$ and $\lambda_{n}$ are non-negative penalization tuning parameters. The BAR estimator of $\boldsymbol{\beta}$ is defined as the limit of this iterative algorithm:

$$
\hat{\boldsymbol{\beta}}=\lim _{k \rightarrow \infty} \hat{\boldsymbol{\beta}}^{(k)}
$$

which can be viewed as a surrogate to $\ell_{0}$-penalized regression.

Note that adaptively reweighting the penalty of a coefficient by the inverse of its squared estimate from the previous iteration allows each coefficient to be penalized differently. At each successive iteration, coefficients whose true values are zero will have larger penalties that will shrink the estimate further towards zero. We have shown in Section S1 of the Online Supplementary Material that the BAR estimator has an oracle property for selection and estimation and a grouping properties for highly correlated covariates.

The BAR estimator can be implemented using the algorithm outlined in Section S2.1 Algorithm S1 of the Online Supplementary Material in which cyclic coordinate decent (CCD) algorithm is employed for each reweighted $\ell_{2}$-penalized regression. Because the algorithm runs a sequence $(k=0,1, \ldots)$ of adaptively reweighted ridge regressions, it adds an extra layer of computational complexity as compared to other popular single-step penalization methods such as LASSO and can create a bottleneck when a large number of iterations is needed. Moreover, because ridge regression is not sparse and thus the limit is never achieved at any given step of the BAR algorithm, an arbitrarily small cutoff value $\epsilon^{*}$ has to be used to induce sparsity in Algorithm S1 (line 18), which is an unpleasant feature. Below we show that these issues can be avoided using a new cyclic BAR algorithm. 


\subsection{A cyclic coordinate-wise BAR algorithm}

In this section, we derive a fast cyclic coordinate-wise BAR algorithm that will result in the elimination of performing multiple ridge regressions and avoid using a cutoff $\epsilon^{*}$ to introduce sparsity as required by the original BAR algorithm (Algorithm S1 in the Online Supplementary Matieral). For a consistent estimate $\tilde{\boldsymbol{\beta}}$ of $\boldsymbol{\beta}$, consider the Cholesky decomposition $-\ddot{l}(\tilde{\boldsymbol{\beta}})=\tilde{\mathbf{X}}^{\prime} \tilde{\mathbf{X}}$ and define $\tilde{\mathbf{y}}=\left(\tilde{\mathbf{X}}^{\prime}\right)^{-1}\{-\ddot{l}(\tilde{\boldsymbol{\beta}}) \tilde{\boldsymbol{\beta}}+\dot{l}(\tilde{\boldsymbol{\beta}})\}$ as the pseudo-response vector. Approximating the negative log-pseudo likelihood by $-l(\boldsymbol{\beta}) \approx(1 / 2)(\tilde{\mathbf{y}}-\tilde{\mathbf{X}} \boldsymbol{\beta})^{\prime}(\tilde{\mathbf{y}}-$ $\tilde{\mathbf{X}} \boldsymbol{\beta})$ using a second-order Taylor expansion in (6) leads to the following solution

$$
\hat{\boldsymbol{\beta}}^{(k)}=g\left(\hat{\boldsymbol{\beta}}^{(k-1)}\right),
$$

where $g(\boldsymbol{\beta})=\left\{\tilde{\mathbf{X}}^{\prime} \tilde{\mathbf{X}}+\lambda_{n} D(\boldsymbol{\beta})\right\}^{-1} \tilde{\mathbf{X}}^{\prime} \tilde{\mathbf{y}}$. and $D(\boldsymbol{\beta})=\operatorname{diag}\left(\beta_{1}^{-2}, \ldots, \beta_{p_{n}}^{-2}\right)$. Hence, as $k \rightarrow \infty$, the limit of the sequence $\left\{\hat{\boldsymbol{\beta}}^{(k)}\right\}$ is the fixed point of the function $g(\cdot)$ or the solution of $g(\boldsymbol{\beta})=\boldsymbol{\beta}$.

The next theorem shows that each component of the fixed-point solution of $g$ can be expressed as a function of all other components. The proof is deferred to Section S1.5 of the Online Supplementary Material.

Theorem 1 Let $\hat{\boldsymbol{\beta}}$ be the fixed-point solution of $g(\cdot)$. Then, for each $j=1, \ldots, p_{n}$, the $j$ th component of $\hat{\boldsymbol{\beta}}$ can be expressed as follows

$$
\hat{\beta}_{j}=g_{j}\left(\hat{\boldsymbol{\beta}}_{-j}\right) \equiv \begin{cases}0, & \text { if }\left|b_{j}\right|<2 \sqrt{\lambda_{n} \tilde{\mathbf{x}}_{j}^{\prime} \tilde{\mathbf{x}}_{j}}, \\ \frac{b_{j}+\operatorname{sign}\left(b_{j}\right) \sqrt{\left(b_{j}\right)^{2}-4 \lambda_{n} \tilde{\mathbf{x}}_{j}^{\prime} \tilde{\mathbf{x}}_{j}}}{2 \tilde{\mathbf{x}}_{j}^{\prime} \tilde{\mathbf{x}}_{j}}, & \text { otherwise, }\end{cases}
$$

where $b_{j}=\tilde{\mathbf{x}}_{j}^{\prime}\left(\tilde{\mathbf{y}}-\sum_{i \neq j} \tilde{\mathbf{x}}_{i} \hat{\beta}_{i}\right)$ and $\hat{\boldsymbol{\beta}}_{-j}=\left(\hat{\beta}_{1}, \ldots, \hat{\beta}_{j-1}, \hat{\beta}_{j+1}, \ldots, \hat{\beta}_{p_{n}}\right)^{\prime}$.

The above result motivates our cyclic coordinate-wise broken adaptive ridge (CYCBAR) algorithm which performs cyclic coordinate-wise updates for the fixed point of $g(\cdot)$ using 


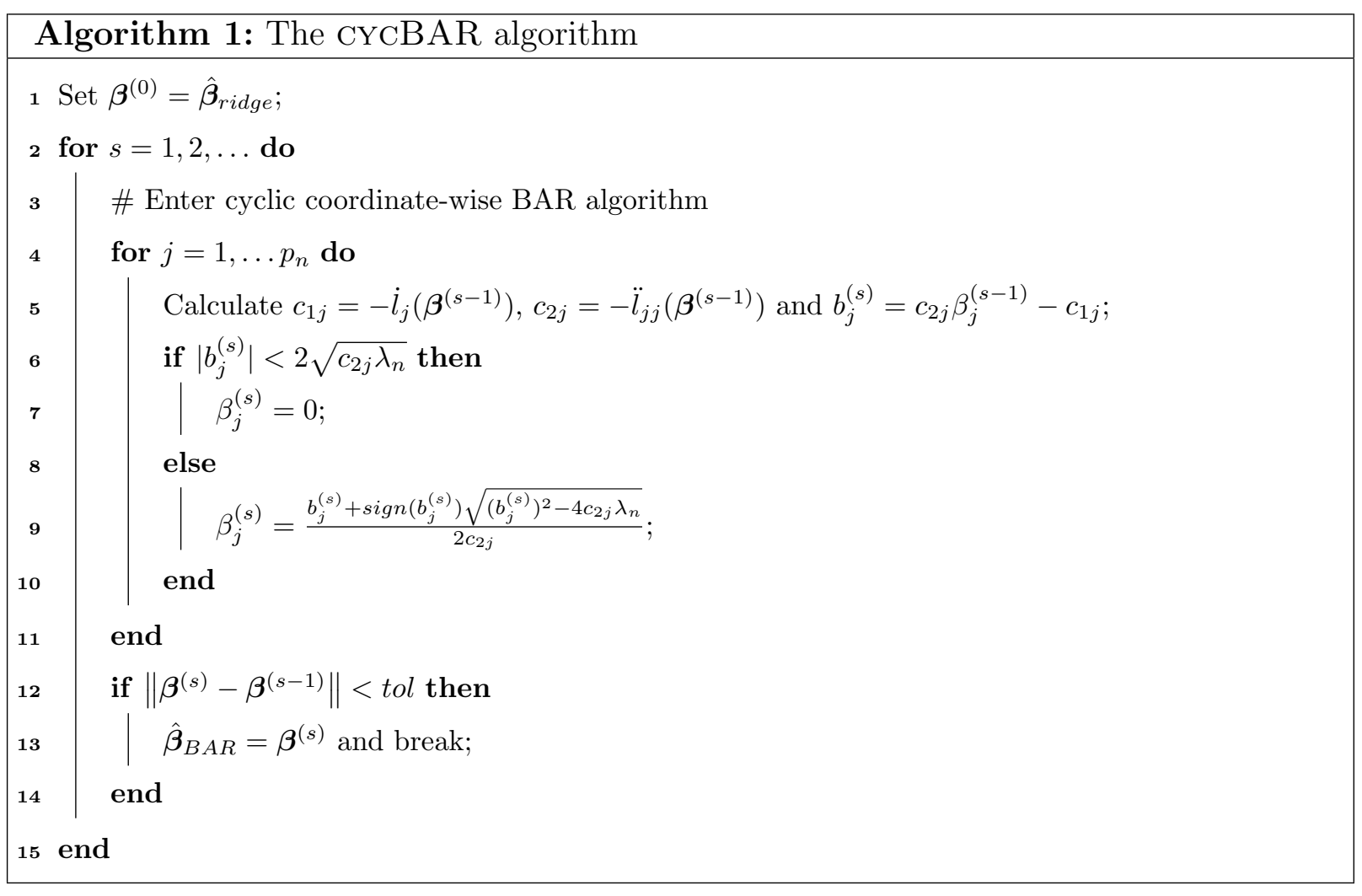

equation (8) as outlined in Algorithm 1 below. In Algorithm 1, $\tilde{\mathbf{X}}$ and $\tilde{\mathbf{y}}$ are initially estimated using the initial ridge estimate $\boldsymbol{\beta}^{(0)}$ and then subsequently updated at step $s$ using the previous estimate $\boldsymbol{\beta}^{(s-1)}$ for $s \geq 1$. Consequently, at step $s$, we have

$$
b_{j}^{(s)} \equiv \tilde{\mathbf{x}}_{j}^{\prime}\left\{\tilde{\mathbf{y}}-\sum_{i \neq j} \tilde{\mathbf{x}}_{i} \beta_{i}^{(s-1)}\right\}=-\ddot{l}_{j j}\left(\boldsymbol{\beta}^{(s-1)}\right) \beta_{j}^{(s-1)}+i_{j}\left(\boldsymbol{\beta}^{(s-1)}\right), \quad \text { for } j=1, \ldots, p_{n},
$$

where $\dot{l}_{j}(\boldsymbol{\beta})$ is the $j$ th element of $-i(\boldsymbol{\beta})$ and $-\ddot{l}_{j j}(\boldsymbol{\beta})$ is the $j$ th diagonal element of $\ddot{l}(\boldsymbol{\beta})$.

Remark 2.1 (CYCBAR versus BAR) The CYCBAR algorithm is derived by approximating the log-psuedo likelihood with a quadratic approximation, so it provides an approximation of the BAR estimator. Because the quadratic approximation is updated iteratively 
in the algorithm, the difference between them are expected to be mostly negligible, which has been corroborated by our empirical studies.

Remark 2.2 (Convergence of CYCBAR) The CYCBAR algorithm resembles the wellknown cyclic coordinate decent (CCD) algorithm that has been commonly used for some popular single-step penalized regression methods such as LASSO. However, its numerical convergence is guaranteed by a different mechanism since the CYCBAR algorithm makes coordinate-wise updates for a fixed-point problem whereas CCD aims to decrease an objective function with each coordinate update. Some graphical illustrations of the convergence of the CYCBAR algorithm for $p_{n}=2$ are given in Section S2.2 Figures S1 and S2 of the Online Supplementary Material. A rigorous proof of the numerical convergence of the CYCBAR algorithm is however not trivial and needs to be investigated in future research.

\subsection{Scalable parameter estimation via forward-backward scan}

Before proceeding further, we note that for the Cox proportional hazards model with no competing risks, $R_{i}=\left\{y: X_{y} \geq X_{i}\right\}$ and $\tilde{w}_{i k} \equiv 1$ for all $i$ and $k$. Therefore the score function can be written as

$$
\dot{l}_{j}(\boldsymbol{\beta})=\sum_{i=1}^{n} I\left(\delta_{i}=1\right) z_{i j}-\sum_{i=1}^{n} I\left(\delta_{i}=1\right) \frac{\sum_{k \in R_{i}} z_{k j} \exp \left(\eta_{k}\right)}{\sum_{k \in R_{i}} \exp \left(\eta_{k}\right)}
$$

for $j=1, \ldots, p_{n}$. Again, if done directly, calculating $i_{j}(\boldsymbol{\beta})$ will require $O\left(n^{2}\right)$ calculations. Suchard et al. (2013) and Mittal et al. (2014), among others, have implemented the following technique to calculate $(9)$ in $O(n)$ calculations. Note that if the event times are arranged in decreasing order, both $\sum_{k \in R_{i}} z_{k j} \exp \left(\eta_{k}\right)$ and $\sum_{k \in R_{i}} \exp \left(\eta_{k}\right)$ are a series of cumulative sums. For example, given $X_{i}>X_{i^{\prime}}$, the set $R_{i^{\prime}}$ consists of the observations from $R_{i}$ and the set of observations $\left\{y: X_{y} \in\left[X_{i^{\prime}}, X_{i}\right)\right\}$, therefore $\sum_{k \in R_{i^{\prime}}} z_{k j} \exp \left(\eta_{k}\right)=$ 
$\sum_{k \in R_{i}} z_{k j} \exp \left(\eta_{k}\right)+\sum_{k \in\left\{y: X_{y} \in\left[X_{i^{\prime}}, X_{i}\right)\right\}} z_{k j} \exp \left(\eta_{k}\right)$ and calculating both $\sum_{k \in R_{i}} z_{k j} \exp \left(\eta_{k}\right)$ and $\sum_{k \in R_{i}} \exp \left(\eta_{k}\right)$, and consequently its ratio, for all $i=1, \ldots, n$ will only require $O(n)$ calculations in total. Furthermore, the outer summation of subjects who observe the event of interest is also a cumulative sum since, provided that $X_{i}>X_{i^{\prime}}$ and both $\delta_{i}=1$ and $\delta_{i^{\prime}}=1$,

$$
\begin{aligned}
\sum_{l=1}^{i} I\left(\delta_{l}=1\right) \frac{\sum_{k \in R_{l}} z_{k j} \exp \left(\eta_{k}\right)}{\sum_{k \in R_{l}} \exp \left(\eta_{k}\right)} & =\sum_{l=1}^{i^{\prime}} I\left(\delta_{l}=1\right) \frac{\sum_{k \in R_{l}} z_{k j} \exp \left(\eta_{k}\right)}{\sum_{k \in R_{l}} \exp \left(\eta_{k}\right)} \\
& +I\left(\delta_{i}=1\right) \frac{\sum_{k \in R_{i}} z_{k j} \exp \left(\eta_{k}\right)}{\sum_{k \in R_{i}} \exp \left(\eta_{k}\right)}
\end{aligned}
$$

which will also only require $O(n)$ calculations since the ratio can be precomputed in $O(n)$ calculations. The diagonal elements of the Hessian also follow a similar derivation and can be calculated in $O(n)$ calculations.

For the PSH model, however, $\sum_{k \in R_{i}} \tilde{w}_{i k} \exp \left(\eta_{j}\right), i=1, \ldots, n$, are not a series of simple cumulative sums because 1 ) the risk sets $R_{i}$ are not monotone over time, and 2) for each $i$, a different set of weights $\tilde{w}_{i k}=\hat{G}\left(X_{i}\right) / \hat{G}\left(X_{i} \wedge X_{k}\right), k \in R_{i}$ are required. To overcome this problem, we show in Lemma 1 below that $\sum_{k \in R_{i}} \tilde{w}_{i k} \exp \left(\eta_{j}\right)$ can be decomposed into a forward cumulative sum and a backward cumulative sum over two disjoint monotone sets. A simple proof is provided in Section S1.6 of the Online Supplementary Material.

Lemma 1 Assume that no ties are present. Then

$$
\sum_{k \in R_{i}} \tilde{w}_{i k} \exp \left(\eta_{k}\right)=\sum_{k \in R_{i}(1)} \exp \left(\eta_{k}\right)+\hat{G}\left(X_{i}\right) \sum_{k \in R_{i}(2)} \exp \left(\eta_{k}\right) / \hat{G}\left(X_{k}\right)
$$

where $R_{i}(1)=\left\{y:\left(X_{y} \geq X_{i}\right)\right\}$ and $R_{i}(2)=\left\{y:\left(X_{y}<X_{i} \cap \epsilon_{y}=2\right)\right\}$ are distinct partitions of $R_{i}$. Furthermore, $R_{i}(1)$ is monotonically decreasing over time and $R_{i}(2)$ is monotonically increasing over time.

Because $R_{i}(1)$ grows cumulatively as the event times decrease from the largest to the smallest, whereas $R_{i}(2)$ grows cumulatively as the observed event times increase from the 
smallest to the largest since it only involves subjects who observed a competing risk and had an observed event time smaller than subject $i$. Thus, similar to the Cox model, the ratio of summations for the score and diagonal Hessian values can be calculated in linear time via a forward-backward scan where one scan goes in one direction to calculate the cumulative sums associated with $R_{i}(1)$ and the other scan goes in the opposite direction to calculate the cumulative sum associated with $R_{i}(2)$. Therefore, we can effectively reduce the number of operations from $O\left(n^{2}\right)$ to $O(n)$.

\section{Simulation study}

\subsection{Simulation setup}

We simulate datasets under various sample sizes and parameter dimensions. The design matrix, $\mathbf{Z}$ was generated from a $p_{n}$-dimensional standard normal distribution with mean zero and pairwise correlation $\operatorname{corr}\left(z_{i}, z_{j}\right)=\rho^{|i-j|}$, where $\rho=0.5$ simulates moderate correlation. The vector of regression parameters for cause 1, the cause of interest, is $\boldsymbol{\beta}_{1}=\left(0.40,0.45,0,0.50,0,0.60,0.75,0,0,0.80, \mathbf{0}_{p-10}\right)$. The data generation scheme follows a similar design to that of Fine and Gray (1999) and Fu et al. (2017). The CIF for cause 1 is $F_{1}\left(t ; \mathbf{z}_{i}\right)=\operatorname{Pr}\left(T_{i} \leq t, \epsilon_{i}=1 \mid \mathbf{z}_{i}\right)=1-[1-\pi\{1-\exp (-t)\}]^{\exp \left(\mathbf{z}_{i}^{\prime} \boldsymbol{\beta}_{1}\right)}$, which is a unit exponential mixture with mass $1-\pi$ at $\infty$ when $\mathbf{z}_{i}=\mathbf{0}$. Unless otherwise noted, the value of $\pi$ is set to 0.5 , which corresponds to a cause 1 event rate of approximately $41 \%$. The CIF for cause 2 is obtained by setting $\operatorname{Pr}\left(\epsilon_{i}=2 \mid \mathbf{z}_{i}\right)=1-\operatorname{Pr}\left(\epsilon_{i}=1 \mid \mathbf{z}_{i}\right)$ and then using an exponential distribution with rate $\exp \left(\mathbf{z}_{i}^{\prime} \boldsymbol{\beta}_{2}\right)$ for the conditional $\operatorname{CIF} \operatorname{Pr}\left(T_{i} \leq t \mid \epsilon_{i}=2, \mathbf{z}_{i}\right)$ with $\boldsymbol{\beta}_{2}=-\boldsymbol{\beta}_{1}$. Censoring times are independently generated from a uniform distribution $U\left(0, u_{\max }\right)$ where $u_{\max }$ controls the censoring percentage. The average censoring percentage for our simulations vary between $30-35 \%$. 


\subsection{Finite-sample properties of BAR}

In this section, we briefly summarize the results for comparing the operating characteristics of BAR along with LASSO (Tibshirani, 1996), SCAD (Fan and Li, 2001), adaptive LASSO (Zou, 2006, ALASSO), and MCP (Zhang, 2010) which are implemented in the crrp package (Fu et al., 2017). Our simulations illustrate that 1) the BAR estimator is insensitive over the choice of $\xi_{n}$ over a large interval and 2) BAR performs as well as other oraclebased procedures in terms of estimation and variable selection. This has been observed consistently over several different combinations of model dimension, event rates, signal values, sample sizes, and model sparsity. We refer readers to Section S3 of the Online Supplementary Material for a more detailed explanation of the conclusions from the study.

\subsection{Computational savings via cycBAR and forward-backward scan}

In this simulation we illustrate the impressive computational savings obtained from $\mathrm{CY}$ CBAR and the forward-backward scan described in Sections 2.3 and 2.4 . We compare three implementations of BAR for the PSH model: the original BAR without the forwardbackward scan, CYCBAR without the forward-backward scan, and CYCBAR with the forward-backward scan. We let $n$ vary from 600 to $2000, p_{n}=100$, and $\rho=0.5$ and compute the runtime of each method averaged over 100 simulations. We report the runtime on a system with an Intel Core i5 $2.9 \mathrm{GHz}$ processor and 16GB of memory.

Figure 1(a) displays the mean runtime (in seconds) for each method as the sample size increases, which shows that the runtime of the original BAR increases quickly while the runtime of BAR implementing both CYCBAR and forward-backward scan grows at a much slower rate. Panels (b) and (c) further demonstrate the separate contributions of CYCBAR 


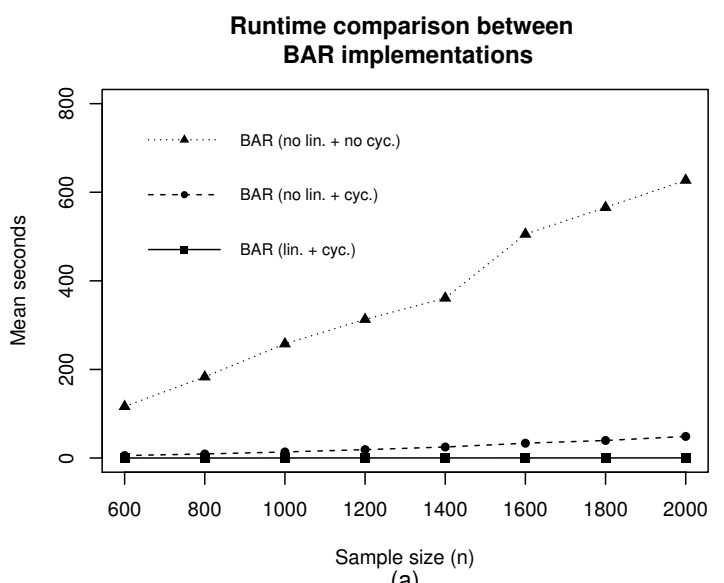

(a)

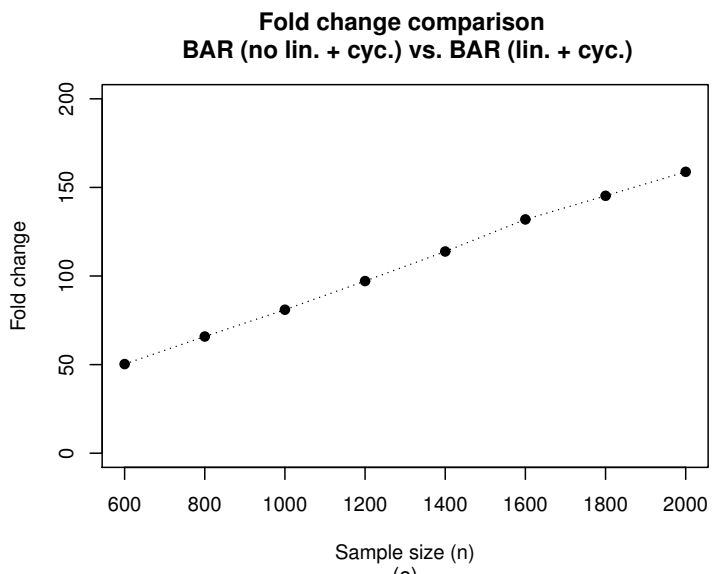

(c)

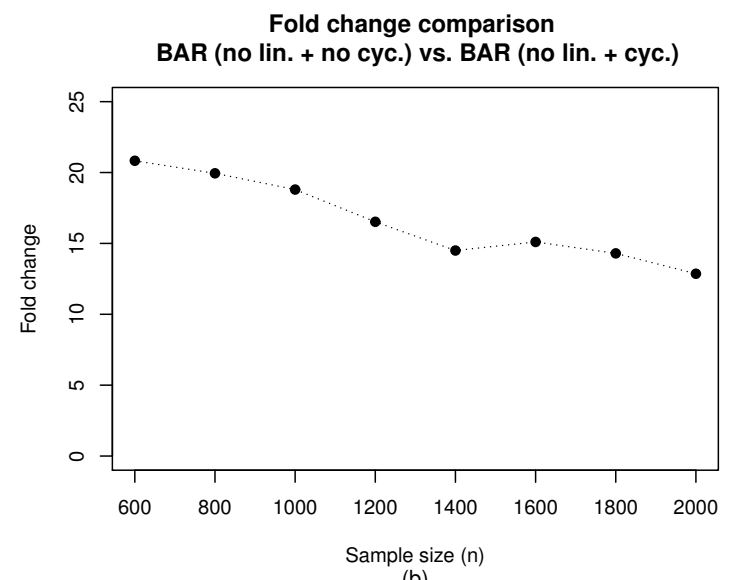

(b)

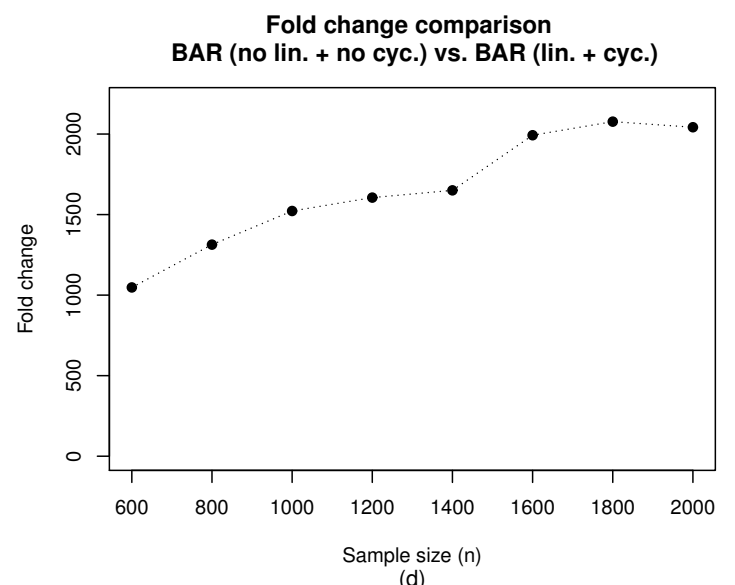

(d)

Figure 1: Runtime comparison between three $\operatorname{BAR}\left(\lambda_{n}\right)$ implementations (cyc. $=$ CYCBAR described in Section 2.3; lin. = forward-backward scan described in Section 2.4.

and the forward-backward scan method, respectively, using fold change. Panel (b) shows a 15-20 fold decrease in runtime between CYCBAR and the original BAR. Panel (c) shows the benefit of linearized estimation, with a 50-150 fold decrease in runtime between CYCBAR with and without the forward-backward scan. Additionally, we perform both SCAD and MCP penalizations both with and without the forward-backward scan implementation and observe similar fold changes as observed in Figure 1(c) and the results are tabulated in Table 
S4 of the Online Supplementary Material. Panel (d) illustrates that using both CYCBAR and the forward-backward scan results in a multiplicative gain, yielding an impressive 1,000-2,000 fold speedup in runtime. The runtime reduction is expected to increase as $n$ and/or $p_{n}$ grow larger as illustrated by the real data example in the following section.

\section{End-stage renal disease}

The United States Renal Data System (USRDS) is a national data system that collects information about end-stage renal disease in the United States. Patients with end-stage renal disease are known to have a shorter life expectancy compared to their disease-free peers (USRDS Annual Report 2017) and kidney transplantation has been shown to provide better health outcomes for patients with end-stage renal disease (Wolfe et al., 1999 , Purnell et al. 2016). As an illustration of the scalability of various methods for large data, we run penalized regressions for a PSH model with 63 demographic and clinical variables using a subset of $n=225,000$ patients from the USRDS that spans a 10-year study time between January 2005 to June 2015. The event of interest was first kidney transplant for patients who were currently on dialysis. Death, renal function recovery, and discontinuation of dialysis are competing risks. Subjects who are lost to follow up or had no event by the end of study period are considered as right censored. We randomly split the data into a training set $(n=125,000)$ and test set $(n=100,000)$. Table S5 in the Online Supplementary Material shows that the proportions of each type of event are similar across the training and test sets.

The BAR method along with SCAD and MCP penalizations are used to fit the PSH

model using the training set. As with Section 3.3, we consider four implementations of BAR: 1) without both CYCBAR and the forward-backward scan; 2) without CYCBAR 
and with the forward-backward scan; 3) with CYCBAR and without the forward-backward scan; and 4) with both CYCBAR and the forward-backward scan. BIC score minimization, implemented with a 25 -value grid search, is used to find the optimal value for the tuning parameter for all three methods. We fix $\xi_{n}=\log \left(p_{n}\right)$ for the BAR method. SCAD and MCP were performed using the crrp R package (Fu et al., 2017) where its generalized cross validation estimation component is removed to allow a fair comparison of their runtime with BAR only for parameter estimation. Additionally, we run SCAD and MCP penalizations using our forward-backward scan to compare the computational performance of our new implementation to the current state of the art. The BIC score based on the training data is used to compare selection performance between models and predictive performance is measured by the concordance index (c-index) proposed by Wolbers et al. (2009) based on the data. Table 1 summarizes the computational time (in seconds), the BIC score, the c-index, and the number of selected variables for each method.

We observe from Table 1 that CYCBAR, without the forward-backward scan, took 46 hours to finish, a marked reduction in runtime over the original BAR implementation which did not finish after 96 hours and was terminated. More impressively, adding the forwardbackward scan resulted in an enormous boost in speeding up the computation, performing the same task in 40 seconds. We observe similar trends in both SCAD and MCP implementations as well. Our forward-backward scan algorithm results in significant reduction in runtime, over-thousand fold, for BAR, SCAD, and MCP, allowing us to perform variable selection for large-scale competing risks data within seconds rather than days.

The predictive and selection performances of all methods are comparable with similar BIC scores, c-index values and model sizes (number of selected variables), that we attribute to the massive sample size of both the training and test set. The variables selection by BAR are also a subset of the variables selected by both SCAD and MCP. Many of the selected 
Table 1: Analysis results of a USRDS data using BAR and CYCBAR along with MCP and SCAD. (BAR/CYCBAR: $\xi_{n}=\log \left(p_{n}\right)$ and $\lambda_{n}$ selected through a grid search; BIC was used to select tuning parameters for all methods; Seconds: Runtime in seconds without the forward-backward scan (no scan) and with (scan); BIC score: BIC score based on the training data; $c$-index: $c$-index based on the test data; Model size: Number of nonzero parameters; *The original BAR without CYCBAR and forward-backward scan did not finish after 96 hours.)

\begin{tabular}{r|rrrr}
\hline \hline & BAR & CYCBAR & SCAD & MCP \\
\hline Seconds (no scan) & $345,600+^{*}$ & 167,020 & 92,571 & 102,565 \\
Seconds (scan) & 1,401 & 40 & 37 & 35 \\
BIC score & 251873.7 & 251867.6 & 251929.9 & 251895.3 \\
$c$-index & 0.85 & 0.85 & 0.85 & 0.85 \\
Model size & 43 & 42 & 48 & 49 \\
\hline
\end{tabular}

variables have been previously reported to have an impact on kidney transplantation.

\section{Discussion}

In extending the $\ell_{0}$-based BAR methodology to the Fine and Gray (1999) PSH model for competing risks data, we have developed a novel coordinate-wise update (CYCBAR) algorithm to avoid carrying out multiple ridge regressions in the original BAR implementation. Furthermore, we introduce a forward-backward scan algorithm to reduce the computational cost of the log-pseudo likelihood and its derivatives for the PSH model from the order of $O\left(n^{2}\right)$ to $O(n)$. While showing comparable selection and estimation performance, the BAR method for the PSH model using the two new algorithms can produce greater than 1,000 
fold speedups over some current penalization methods for the PSH model in numerical studies.

An important domain of application of the developed scalable sparse regression method is large comparative effectiveness and drug safety studies using massive electronic health record (EHR) databases such as the Observational Health Data Sciences and Informatics (OHDSI) program (Hripcsak et al., 2015) (https://ohdsi.org/) and the U.S. FDA's Sentinel Initiative (https://www.fda.gov/safety/fdassentinelinitiative/ucm2007250.htm). These massive databases typically contain millions to hundreds of millions patient records with tens of thousands patient attributes, which are particularly useful for drug safety studies of a rare event (such as an unexpected severe adverse event (SAE)) to protect public health.

As illustrated by the USRDS data example in Section 4, while existing methods for the PSH model is likely to grind to a halt, the developed scalable BAR method with highperformance algorithms has made it possible to analyze these massive data in real time. To this end, we point out that for a large data with millions of patient records on tens of thousands covariates, it may not always be feasible to fit a model when the data is stored in the standard dense format due to the high memory requirement. On the other hand, these massive datasets are often sparse with only a small portion of covariates are being nonzeros for a given subject. We are currently working on implementing the developed BAR method for sparse massive sample size competing risks data by exploiting the sparsity in the data matrix.

Finally, we emphasize that the developed CYCBAR method in Section 2.3 and the forward-backward scan method of Lemma 1 in Section 2.4 are of interest on their own. The CYCBAR method can be applied directly to other models and data settings. It is also straightforward to apply the forward-backward scan method to accelerate other estimation methods for the PSH model. Using this approach, we are currently developing a stand- 
alone package for $\mathrm{R}$ that includes the unpenalized estimation method of Fine and Gray (1999) and other popular penalization methods.

\section{Acknowledgement}

The manuscript was reviewed and approved for publication by an officer of the National Institute of Diabetes and Digestive and Kidney Diseases. Data reported herein were supplied by the USRDS. Interpretation and reporting of these data are the responsibility of the authors and in no way should be seen as official policy or interpretation of the US government. Marc A. Suchard's work is partially supported through the National Institute of Health grant U19 AI 135995. The research of Gang Li was partly supported by National Institute of Health Grants P30 CA-16042, UL1TR000124-02, and P50 CA211015.

\section{References}

Ahn, K. W., A. Banerjee, N. Sahr, and S. Kim (2018). Group and within-group variable selection for competing risks data. Lifetime Data Analysis 24(3), 407-424.

Candes, E. J., M. B. Wakin, and S. P. Boyd (2008). Enhancing sparsity by reweighted 11 minimization. Journal of Fourier Analysis and Applications 14 (5), 877-905.

Chartrand, R. and W. Yin (2008). Iterative reweighted algorithms for compressive sensing. In 2008 IEEE International Conference on Acoustics, Speech and Signal Processing.

Cho, H. and A. Qu (2013). Model selection for correlated data with diverging number of parameters. Statistica Sinica 26, 901-927. 
Cox, D. R. (1972). Regression models and life-tables. Journal of the Royal Statistical Society: Series B (Statistical Methodology) 34(2), 187-220.

Dai, L., K. Chen, Z. Sun, Z. Liu, and G. Li (2018). Broken adaptive ridge regression and its asymptotic properties. Journal of Multivariate Analysis 168, 334-351.

Daubechies, I., R. DeVore, M. Fornasier, and C. S. Güntürk (2010). Iteratively reweighted least squares minimization for sparse recovery. Communications on Pure and Applied Mathematics 63(1), 1-38.

Fan, J. and R. Li (2001). Variable selection via nonconcave penalized likelihood and its oracle properties. Journal of the American Statistical Association 96(456), 1348-1360.

Fan, J., H. Peng, et al. (2004). Nonconcave penalized likelihood with a diverging number of parameters. The Annals of Statistics 32(3), 928-961.

Fine, J. P. and R. J. Gray (1999). A proportional hazards model for the subdistribution of a competing risk. Journal of the American Statistical Association 94(446), 496-509.

Fu, Z., C. R. Parikh, and B. Zhou (2017). Penalized variable selection in competing risks regression. Lifetime Data Analysis 23(3), 353-376.

Gasso, G., A. Rakotomamonjy, and S. Canu (2009). Recovering sparse signals with a certain family of nonconvex penalties and dc programming. IEEE Transactions on Signal Processing 57(12), 4686-4698.

Gorodnitsky, I. F. and B. D. Rao (1997). Sparse signal reconstruction from limited data using focuss: A re-weighted minimum norm algorithm. IEEE Transactions on Signal Processing 45(3), 600-616. 
Gray, R. J. (1988). A class of k-sample tests for comparing the cumulative incidence of a competing risk. The Annals of Statistics 16(3), 1141-1154.

Ha, I. D., M. Lee, S. Oh, J.-H. Jeong, R. Sylvester, and Y. Lee (2014). Variable selection in subdistribution hazard frailty models with competing risks data. Statistics in Medicine 33(26), 4590-4604.

Hou, J., A. Paravati, J. Hou, R. Xu, and J. Murphy (2018). High-dimensional variable selection and prediction under competing risks with application to seer-medicare linked data. Statistics in Medicine 37(24), 3486-3502.

Hripcsak, G., J. D. Duke, N. H. Shah, C. G. Reich, V. Huser, M. J. Schuemie, M. A. Suchard, R. W. Park, I. C. K. Wong, P. R. Rijnbeek, et al. (2015). Observational health data sciences and informatics (ohdsi): opportunities for observational researchers. Studies in Health Technology and Informatics 216, 574-578.

Kaplan, E. L. and P. Meier (1958). Nonparametric estimation from incomplete observations. Journal of the American Statistical Association 53(282), 457-481.

Kosorok, M. R. and S. Ma (2007). Marginal asymptotics for the "large p, small n" paradigm: With applications to microarray data. The Annals of Statistics 35(4), 1456-1468.

Mittal, S., D. Madigan, R. S. Burd, and M. A. Suchard (2014). High-dimensional, massive sample-size cox proportional hazards regression for survival analysis. Biostatistics 15(2), $207-221$.

Ni, A., J. Cai, and D. Zeng (2016). Variable selection for case-cohort studies with failure time outcome. Biometrika $103(3), 547-562$.

Pintilie, M. (2006). Competing Risks: A Practical Perspective. John Wiley \& Sons. 
Prentice, R., J. Kalbfleisch, A. Peterson Jr, N. Flournoy, V. Farewell, and N. Breslow (1978). The analysis of failure times in the presence of competing risks. Biometrics 34(4), $541-554$.

Purnell, T. S., X. Luo, L. M. Kucirka, L. A. Cooper, D. C. Crews, A. B. Massie, L. E. Boulware, and D. L. Segev (2016). Reduced racial disparity in kidney transplant outcomes in the united states from 1990 to 2012. Journal of the American Society of Nephrology, ASN-2015030293.

Putter, H., M. Fiocco, and R. Geskus (2007). Tutorial in biostatistics: competing risks and multi-state models. Statistics in Medicine 26(11), 2389-2430.

Schuemie, M. J., P. B. Ryan, D. Hripcsak, George Madigan, and M. A. Suchard (2017). Honest learning for the healthcare system: large-scale evidence from real-world data. Science Under review.

Suchard, M. A., S. E. Simpson, I. Zorych, P. Ryan, and D. Madigan (2013). Massive parallelization of serial inference algorithms for a complex generalized linear model. ACM Transactions on Modeling and Computer Simulation 23(1).

Tibshirani, R. (1996). Regression shrinkage and selection via the lasso. Journal of the Royal Statistical Society: Series B (Statistical Methodology) 58(1), 267-288.

Wipf, D. and S. Nagarajan (2010). Iterative reweighted $\ell_{1}$ and $\ell_{2}$ methods for finding sparse solutions. IEEE Journal of Selected Topics in Signal Processing 4(2), 317-329.

Wolbers, M., M. T. Koller, J. C. Witteman, and E. W. Steyerberg (2009). Prognostic models with competing risks: methods and application to coronary risk prediction. Epidemiology 20(4), 555-561. 
Wolfe, R. A., V. B. Ashby, E. L. Milford, A. O. Ojo, R. E. Ettenger, L. Y. Agodoa, P. J. Held, and F. K. Port (1999). Comparison of mortality in all patients on dialysis, patients on dialysis awaiting transplantation, and recipients of a first cadaveric transplant. New England Journal of Medicine 341(23), 1725-1730.

Zhang, C.-H. (2010). Nearly unbiased variable selection under minimax concave penalty. The Annals of Statistics 38(2), 894-942.

Zhang, X. and G. Cheng (2017). Simultaneous inference for high-dimensional linear models. Journal of the American Statistical Association 112(518), 757-768.

Zhao, H., D. Sun, G. Li, and J. Sun (2018). Variable selection for recurrent event data with broken adaptive ridge regression. Canadian Journal of Statistics https://doi.org/10.1002/cjs.11459.

Zhao, H., D. Sun, G. Li, and J. Sun (2019). Simultaneous estimation and variable selection for incomplete event history studies. Journal of Multivariate Analysis 171, 359-361.

Zhao, H., Q. Wu, G. Li, and J. Sun (2019). Simultaneous estimation and variable selection for interval-censored data with broken adaptive ridge regression. Journal of the American Statistical Association 0(0), 1-13.

Zou, H. (2006). The adaptive lasso and its oracle properties. Journal of the American statistical association 101(476), 1418-1429. 


\section{ONLINE SUPPLEMENTARY MATERIAL \\ for "Scalable Algorithms for Large Competing Risks Data"}

\section{A Statements and technical proofs of theorems and lemmas}

\section{A.1 Regularity conditions}

Define

$$
\begin{aligned}
S^{(k)}(\boldsymbol{\beta}, s) & =\frac{1}{n} \sum_{i=1}^{n} \hat{w}_{i}(s) Y_{i}(s) \mathbf{z}_{i}^{\otimes k} \exp \left(\boldsymbol{\beta}^{\prime} \mathbf{z}_{i}\right), \quad k=0,1,2, \\
\mathbf{E}(\boldsymbol{\beta}, s) & =S^{(1)}(\boldsymbol{\beta}, s) / S^{(0)}(\boldsymbol{\beta}, s), \\
\text { and } & \\
V(\boldsymbol{\beta}, s) & =S^{(2)}(\boldsymbol{\beta}, s) / S^{(0)}(\boldsymbol{\beta}, s)-\mathbf{E}(\boldsymbol{\beta}, s)^{\otimes 2}
\end{aligned}
$$

where $\mathbf{x}^{\otimes k}=\left(1, \mathbf{x}, \mathbf{x x}^{\prime}\right)$ for $k=0,1,2$, respectively. Moreover, with $N_{i}(t)=I\left(T_{i} \leq t, \epsilon_{i}=1\right)$ and $Y_{i}(t)=1-N_{i}(t-)$ define $M_{i}(\boldsymbol{\beta}, t)=\int_{0}^{t} d N_{i}(u)-\int_{0}^{t} Y_{i}(u) h_{10}(u) \exp \left(\boldsymbol{\beta}^{\prime} \mathbf{z}_{i}\right) d u$. Similarly, with defining $N_{i}^{c}(t)=I\left(C_{i} \leq t\right)$ and $H^{c}(t)$ being the cumulative hazard function by treating the censored observations as events, $M_{i}^{c}(t)=N_{i}^{c}(t)-\int_{0}^{t} I\left(X_{i} \geq u\right) d H^{c}(u)$. Let $\|\cdot\|_{p}$ be the $\ell_{p}$-norm for vectors and the norm induced by the vector $p_{n}$-norm for matrices. The following technical conditions will be needed in our derivations for the statistical properties of the pshBAR estimator.

(C1) $\int_{0}^{\tau} h_{01}(t) d t<\infty$;

(C2) There exists some compact neighborhood, $\mathcal{B}_{0}$, of the true value $\boldsymbol{\beta}_{0}$ such that for $k=0,1,2$, there exists a scalar, vector, and matrix function $s^{(k)}(\boldsymbol{\beta}, t)$ defined on 
$\mathcal{B}_{0} \times[0, \tau]$ such that

$$
\sup _{t \in[0, \tau], \boldsymbol{\beta} \in \mathcal{B}_{0}}\left\|S^{(k)}(\boldsymbol{\beta}, t)-s^{(k)}(\boldsymbol{\beta}, t)\right\|_{2}=o_{p}(1), \quad \text { as } n \rightarrow \infty
$$

(C3) Let $s^{(1)}(\boldsymbol{\beta}, t)=\partial s^{(0)}(\boldsymbol{\beta}, t) / \partial \boldsymbol{\beta}$ and $s^{(2)}(\boldsymbol{\beta}, t)=\partial s^{(1)}(\boldsymbol{\beta}, t) / \partial \boldsymbol{\beta}$. For $k=0,1,2$, the functions $s^{(k)}(\boldsymbol{\beta}, t)$ are continuous with respect to $\boldsymbol{\beta} \in \mathcal{B}_{0}$, uniformly in $t \in[0, \tau]$, and are bounded on $\boldsymbol{\beta}_{0} \times[0, \tau]$; furthermore, $s^{(0)}(\boldsymbol{\beta}, t)$ is bounded away from zero on $\mathcal{B}_{0} \times[0, \tau]$

(C4) Let $\mathbf{e}(\boldsymbol{\beta}, t)=s^{(1)}(\boldsymbol{\beta}, t) / s^{(0)}(\boldsymbol{\beta}, t), v(\boldsymbol{\beta}, t)=s^{(2)}(\boldsymbol{\beta}, t) / s^{(0)}(\boldsymbol{\beta}, t)-\mathbf{e}(\boldsymbol{\beta}, t)^{\otimes 2}$, and $\Omega=\int_{0}^{\tau} v\left(\boldsymbol{\beta}_{0}, u\right) s^{(0)}\left(\boldsymbol{\beta}_{0}, u\right) h_{10}(u) d u$. There exists some constants $C_{2}$ and $C_{3}$ such that

$$
0<C_{2}<\text { eigen }_{\min }(\Omega) \leq \text { eigen }_{\max }(\Omega)<C_{3}<\infty
$$

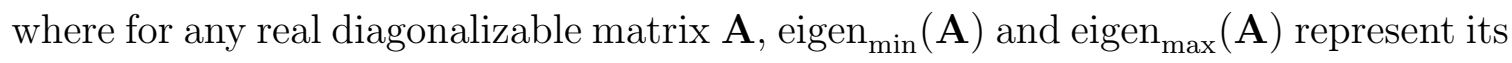
smallest and largest eigenvalues, respectively; furthermore, there also exists a matrix $\Gamma$ such that $\left\|n^{-1} \sum_{i=1}^{n} \operatorname{var}\left(\mathbf{U}_{i}\right)-\Gamma\right\|_{2} \rightarrow 0$, where

$$
\mathbf{U}_{i}=\int_{0}^{\tau}\left\{\mathbf{z}_{i}(u)-\mathbf{e}\left(\boldsymbol{\beta}_{0}, u\right)\right\} w_{i}(u) d M_{i}\left(\boldsymbol{\beta}_{0}, u\right)+\int_{0}^{\tau} \mathbf{q}(u) / \pi(u) d M_{i}^{c}(u),
$$

for

$$
\begin{aligned}
& w_{i}(t)=I\left(C_{i} \geq T_{i} \wedge t\right) G(t) / G\left(X_{i} \wedge t\right) \\
& \mathbf{q}(u)=-\lim _{n \rightarrow \infty} \frac{1}{n} \sum_{i=1}^{n} \int_{0}^{\tau}\left\{\mathbf{z}_{i}(t)-\mathbf{e}\left(\boldsymbol{\beta}_{0}, t\right)\right\} w_{i}(t) I\left(X_{i}<u \leq t\right) d M_{i}\left(\boldsymbol{\beta}_{0}, t\right) \\
& \pi(u)=\lim _{n \rightarrow \infty} \frac{1}{n} \sum_{i=1}^{n} I\left(X_{i} \geq u\right)
\end{aligned}
$$

(C5) There exists a constant $C_{6}$ such that $\sup _{1 \leq i \leq n} E\left(U_{i j}^{2} U_{i l}^{2}\right)<C_{6}<\infty$ for all $1 \leq j, l \leq p$, where $U_{i j}$ is the $j$-th element of $\mathbf{U}_{i}$ defined in (C4); 
(C6) As $n \rightarrow \infty, p_{n}^{4} / n \rightarrow 0, \lambda_{n} \rightarrow \infty, \xi_{n} \rightarrow \infty, \xi_{n} b_{n} / \sqrt{n} \rightarrow 0, p /\left(n a_{n}^{2}\right) \rightarrow 0, \lambda_{n} b_{n}^{3} \sqrt{q_{n}} / \sqrt{n} \rightarrow$ 0 and $\lambda_{n} \sqrt{q_{n}} /\left(a_{n}^{3} \sqrt{n}\right) \rightarrow 0$, where $a_{n}=\min _{j=1, \ldots, q_{n}}\left(\left|\beta_{0 j}\right|\right)$ and $b_{n}=\max _{j=1, \ldots q_{n}}\left(\left|\beta_{0 j}\right|\right)$.

The above conditions (C1)-(C5) are similar to those proposed by ? and Ahn et al. (2018). Condition (C1) ensures a finite baseline cumulative hazard. Condition (C2) ensures the asymptotic stability of $S^{(k)}(\boldsymbol{\beta}, t)$, as required under fixed dimension. Under diverging dimension, it follows from Theorem 1 of Kosorok and Ma (2007) that under certain regularity conditions, $\sup _{t \in[0,1], \boldsymbol{\beta} \in \mathcal{B}_{0}}\left\|S^{(k)}(\boldsymbol{\beta}, t)-s^{(k)}(\boldsymbol{\beta}, t)\right\|_{2} \leq \sqrt{p_{n} \ln p / n}$, which implies that (C2) holds if $p_{n} \ln p / n \rightarrow 0$. Condition (C3) requires that $\exp \left(\boldsymbol{\beta}^{\prime} \mathbf{z}_{i}\right)$ remain integrable under diverging dimension. This will allow integration and differentiation with respect to $S^{(k)}(\boldsymbol{\beta}, t)(k=0,1)$ to be interchanged in our technical derivations. Condition (C4) guarantees that the covariance matrix of the score function is positive definite and has uniformly bounded eigenvalues for all $n$ and $\boldsymbol{\beta} \in \mathcal{B}_{0}$. Other authors in the variable selection literature have also required a slightly weaker condition (Fan et al., 2004; ? Cho and Qu, 2013; Ni et al., 2016; Ahn et al., 2018). Condition (C5) is vital in proving the Lindeberg condition under diverging dimension for our proof. Condition (C6) specifies the divergence or convergence rates for the model size, the penalty tuning parameters, and the lower and upper bound of the true signal. These technical assumptions are only sufficient conditions for our theoretical derivations and observations from our empirical studies illustrate that our theoretical results may, in fact, hold under weaker conditions. Further, we would like to point out that the conditions in (C6) do not impose any one-to-one relationship in finite scenarios.

Remark A.1 Ahn et al. (2018) showed that under Conditions (C1) - (C5) and $p_{n}^{4} / n \rightarrow 0$

$$
\left\|\dot{i}\left(\boldsymbol{\beta}_{0}\right)\right\|_{2}=O_{p}\left(\sqrt{n p_{n}}\right)
$$


and

$$
n^{-1} \ddot{l}(\boldsymbol{\beta})=\Omega+o_{p}(1)
$$

in probability, uniformly in $\boldsymbol{\beta} \in \mathcal{B}_{0}$.

\section{A.2 Statement and proof of the oracle property}

Let $\boldsymbol{\beta}_{1}$ and $\boldsymbol{\beta}_{2}$ be the first $q_{n}$ and remaining $p_{n}-q_{n}$ components of $\boldsymbol{\beta}$, respectively, and define $\boldsymbol{\beta}_{0}=\left(\boldsymbol{\beta}_{01}^{\prime}, \boldsymbol{\beta}_{02}^{\prime}\right)^{\prime}$ as the true values of $\boldsymbol{\beta}$ where, without loss of generality, $\boldsymbol{\beta}_{01}=$ $\left(\beta_{01} \ldots, \beta_{0 q_{n}}\right)$ is a vector of $q_{n}$ non-zero values and $\boldsymbol{\beta}_{02}=\mathbf{0}$ is a $p_{n}-q_{n}$ dimensional vector of zeros. Below we state the asymptotic properties of the BAR estimator for the PSH model under certain regularity conditions.

Theorem 2 (Oracle property) Assume the regularity conditions (C1) - (C6) in the Section S1.1. Let $\hat{\boldsymbol{\beta}}_{1}$ and $\hat{\boldsymbol{\beta}}_{2}$ be the first $q_{n}$ and the remaining $p_{n}-q_{n}$ components of the $B A R$ estimator $\hat{\boldsymbol{\beta}}$, respectively. Then,

(a) $\hat{\boldsymbol{\beta}}_{2}=\mathbf{0}$ with probability tending to one;

(b) $\sqrt{n} \mathbf{d}_{n}^{\prime} \Gamma_{11}^{-1 / 2} \Omega_{11}\left(\hat{\boldsymbol{\beta}}_{1}-\boldsymbol{\beta}_{01}\right) \rightarrow N(0,1)$, for any $q_{n}$-dimensional vector $\mathbf{d}_{n}$ such that $\left\|\mathbf{d}_{n}\right\|_{2} \leq 1$ and where $\Gamma_{11}$ and $\Omega_{11}$ are the first $q_{n} \times q_{n}$ submatrices of $\Gamma$ and $\Omega$, respectively, defined in Condition (C4).

Theorem 2(a) establishes that with large probability, the true zero coefficients will be estimated as zeros by the BAR estimator. Part (b) of the theorem essentially states that the nonzero component of the BAR estimator is asymptotically normal and equivalent to the weighted ridge estimator of the oracle model. To prove Theorem 2 we first establish four lemmas. 


\section{Lemma 2 (Consistency of ridge estimator) Let}

$$
\hat{\boldsymbol{\beta}}_{\text {ridge }}=\arg \min _{\boldsymbol{\beta}}\left\{-2 l(\boldsymbol{\beta})+\sum_{j=1}^{p_{n}} \xi_{n} \beta_{j}^{2}\right\},
$$

be the PSH ridge estimator defined in Equation (3). Assume that Conditions (C1) - (C6) hold. Then

$$
\left\|\hat{\boldsymbol{\beta}}_{\text {ridge }}-\boldsymbol{\beta}_{0}\right\|_{2}=O_{p}\left[\sqrt{p_{n}}\left\{n^{-1 / 2}\left(1+\xi_{n} b_{n} / \sqrt{n}\right)\right\}\right]=O_{p}\left(\sqrt{p_{n} / n}\right),
$$

where $b_{n}$ is an upper bound of the true nonzero $\left|\beta_{0 j}\right|$ 's defined in Condition (C6).

Proof. Let $\alpha_{n}=\sqrt{p_{n}}\left(n^{-1 / 2}+\xi_{n} b_{n} / n\right)$ and $\ell(\boldsymbol{\beta})=-2 l(\boldsymbol{\beta})+\xi_{n} \sum_{j=1}^{p_{n}} \beta_{j}^{2}$. To prove Lemma 2, it is sufficient to show that for any $\epsilon>0$, there exists a large enough constant $K_{0}$ such that

$$
\operatorname{pr}\left\{\inf _{\|\mathbf{u}\|_{2}=K_{0}} L\left(\boldsymbol{\beta}_{0}+\alpha_{n} \mathbf{u}\right)>L\left(\boldsymbol{\beta}_{0}\right)\right\} \geq 1-\epsilon,
$$

since (16) implies that there exists a local minimum, $\hat{\boldsymbol{\beta}}_{\text {ridge }}$, inside the ball $\left\{\boldsymbol{\beta}_{0}+\alpha_{n} \mathbf{u}\right.$ : $\left.\|\mathbf{u}\|_{2} \leq K_{0}\right\}$ such that $\left\|\hat{\boldsymbol{\beta}}_{\text {ridge }}-\boldsymbol{\beta}_{0}\right\|_{2}=O_{p}\left(\alpha_{n}\right)$, with probability tending to one. To prove (16), we first note

$$
\begin{aligned}
\frac{1}{n} L\left(\boldsymbol{\beta}_{0}+\alpha_{n} \mathbf{u}\right)-\frac{1}{n} L\left(\boldsymbol{\beta}_{0}\right) & =-\frac{1}{n}\left\{2 l\left(\boldsymbol{\beta}_{0}+\alpha_{n} \mathbf{u}\right)-\frac{1}{n} 2 l\left(\boldsymbol{\beta}_{0}\right)\right\}+\frac{\xi_{n}}{n} \sum_{j=1}^{p_{n}}\left\{\left(\beta_{0 j}+\alpha_{n} u_{j}\right)^{2}-\beta_{0 j}^{2}\right\} \\
& =-\frac{1}{n}\left\{2 l\left(\boldsymbol{\beta}_{0}+\alpha_{n} \mathbf{u}\right)-2 l\left(\boldsymbol{\beta}_{0}\right)\right\}+\frac{\xi_{n}}{n} \sum_{j=1}^{p_{n}}\left(2 \beta_{0 j} \alpha_{n} u_{j}+\alpha_{n}^{2} u_{j}^{2}\right) \\
& \geq-\frac{1}{n}\left\{2 l\left(\boldsymbol{\beta}_{0}+\alpha_{n} \mathbf{u}\right)-2 l\left(\boldsymbol{\beta}_{0}\right)\right\}+\frac{2 \xi_{n} \alpha_{n}}{n} \sum_{j=1}^{p_{n}} \beta_{0 j} u_{j} \\
& =-\frac{1}{n}\left\{2 l\left(\boldsymbol{\beta}_{0}+\alpha_{n} \mathbf{u}\right)-2 l\left(\boldsymbol{\beta}_{0}\right)\right\}+\frac{2 \xi_{n} \alpha_{n}}{n} \sum_{j=1}^{q_{n}} \beta_{0 j} u_{j} \\
& \equiv W_{1}+W_{2} .
\end{aligned}
$$


By Taylor expansion, we have

$$
\begin{aligned}
W_{1} & =-\frac{2}{n} \alpha_{n} \mathbf{u}^{\prime} i\left(\boldsymbol{\beta}_{0}\right)-\frac{1}{n} \alpha_{n}^{2} \mathbf{u}^{\prime} \ddot{l}\left(\boldsymbol{\beta}^{*}\right) \mathbf{u} \\
& =W_{11}+W_{12},
\end{aligned}
$$

where $\boldsymbol{\beta}^{*}$ lies between $\boldsymbol{\beta}_{0}$ and $\boldsymbol{\beta}_{0}+\alpha_{n} \mathbf{u}$, and $i(\boldsymbol{\beta})$ and $\ddot{l}(\boldsymbol{\beta})$ denote the first and second derivatives of $l(\boldsymbol{\beta})$, respectively. By the Cauchy-Schwartz inequality,

$$
W_{11}=-\frac{2}{n} \alpha_{n} \mathbf{u}^{\prime} i\left(\boldsymbol{\beta}_{0}\right) \leq \frac{2}{n} \alpha_{n}\left\|i\left(\boldsymbol{\beta}_{0}\right)\right\|_{2} \cdot\|\mathbf{u}\|_{2}=\frac{2}{n} \alpha_{n} O_{p}\left(\sqrt{n p_{n}}\right)\|\mathbf{u}\|_{2} \leq O_{p}\left(\alpha_{n}^{2}\right)\|\mathbf{u}\|_{2},
$$

where the second equality is due to (13). By (14) we have

$$
W_{12}=-\frac{1}{n} \alpha_{n}^{2} \mathbf{u}^{\prime} l\left(\boldsymbol{\beta}^{*}\right) \mathbf{u}=\alpha_{n}^{2} \mathbf{u}^{\prime} \Omega \mathbf{u}\left\{1+o_{p}(1)\right\}
$$

Since eigen $_{\text {min }}(\Omega) \geq C_{2}>0$ by Condition (C4), $W_{12}$ dominates $W_{11}$ uniformly in $\|\mathbf{u}\|_{2}=K_{0}$ for a sufficiently large $K_{0}$. Furthermore

$$
W_{2} \leq \frac{2 \xi_{n} \alpha_{n}}{n}\left|\boldsymbol{\beta}_{01}^{\prime} \mathbf{u}\right| \leq \frac{2 \sqrt{q_{n}} \xi_{n} \alpha_{n} b_{n}}{n}\|\mathbf{u}\|_{2}=O_{p}\left(\alpha_{n}^{2}\right)\|\mathbf{u}\|_{2},
$$

where the last step follows from the fact that $\sqrt{q_{n}} \xi_{n} b_{n} / n<\sqrt{p_{n}}\left(n^{-1 / 2}+\xi_{n} b_{n} / n\right)=\alpha_{n}$. Therefore for a sufficiently large $K_{0}$, we have that $W_{12}$ dominates $W_{11}$ and $W_{2}$ uniformly in $\|\mathbf{u}\|_{2}=K_{0}$. Since $W_{12}$ is positive, 16 holds and therefore $\left\|\hat{\boldsymbol{\beta}}_{\text {ridge }}-\boldsymbol{\beta}_{0}\right\|_{2}=O_{p}\left(\alpha_{n}\right)=$ $O_{p}\left[\sqrt{p_{n}}\left\{n^{-1 / 2}\left(1+\xi_{n} b_{n} / \sqrt{n}\right)\right\}\right]=O_{p}\left(\sqrt{p_{n} / n}\right)$, where the last step follows from condition (C6).

Remark A.2 Recall $\boldsymbol{\beta}=\left(\boldsymbol{\beta}_{1}^{\prime}, \boldsymbol{\beta}_{2}^{\prime}\right)^{\prime}$ where $\boldsymbol{\beta}_{1}^{\prime}$ and $\boldsymbol{\beta}_{2}^{\prime}$ correspond to the first $q_{n}$ and remaining $p_{n}-q$ components of $\boldsymbol{\beta}$, respectively. Let

$$
Q_{n}(\boldsymbol{\theta} \mid \boldsymbol{\beta})=-2 l(\boldsymbol{\theta})+\lambda_{n} \boldsymbol{\theta}^{\prime} D(\boldsymbol{\beta}) \boldsymbol{\theta},
$$


where $D(\boldsymbol{\beta})=\operatorname{diag}\left(\beta_{1}^{-2}, \beta_{2}^{-2}, \ldots, \beta_{q_{n}}^{-2}, \beta_{q_{n}+1}^{-2}, \ldots, \beta_{p_{n}}^{-2}\right)$ and $l(\boldsymbol{\theta})$ is the $p_{n}$-dimensional $\log$ partial likelihood of the reduced model. Let $\dot{Q}(\boldsymbol{\theta} \mid \boldsymbol{\beta})$ and $\ddot{Q}(\boldsymbol{\theta} \mid \boldsymbol{\beta})$ be the first and second derivatives of $Q(\boldsymbol{\theta} \mid \boldsymbol{\beta})$ with respective to $\boldsymbol{\theta}$, respectively. Then

$$
\begin{gathered}
\dot{Q}(\boldsymbol{\theta} \mid \boldsymbol{\beta})=-2 \dot{l}(\boldsymbol{\theta})+2 \lambda_{n} D(\boldsymbol{\beta}) \boldsymbol{\theta}, \\
\ddot{Q}(\boldsymbol{\theta} \mid \boldsymbol{\beta})=-2 \ddot{l}(\boldsymbol{\theta})+2 \lambda_{n} D(\boldsymbol{\beta}) .
\end{gathered}
$$

Remark A.3 Let $\hat{\boldsymbol{\beta}}_{\text {ridge }, 1}$ and $\hat{\boldsymbol{\beta}}_{\text {ridge }, 2}$ denote the first $q_{n}$ and the remaining $p_{n}-q_{n}$ compo-

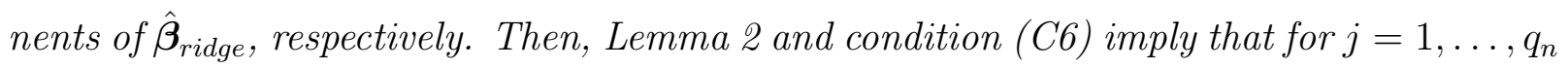
and sufficiently large $n, a_{n} / 2 \leq\left|\hat{\beta}_{\text {ridge }, 1 j}\right| \leq 2 b_{n}$, where $\hat{\beta}_{\text {ridge }, 1 j}$ is the $j^{\text {th }}$ component of $\hat{\boldsymbol{\beta}}_{\text {ridge }, 1}$ and $\left\|\hat{\boldsymbol{\beta}}_{\text {ridge }, 2}\right\|_{2}=O\left(\sqrt{p_{n} / n}\right)$.

Remark A.4 Recall $\boldsymbol{\beta}=\left(\boldsymbol{\beta}_{1}^{\prime}, \boldsymbol{\beta}_{2}^{\prime}\right)^{\prime}$ where $\boldsymbol{\beta}_{1}^{\prime}$ and $\boldsymbol{\beta}_{2}^{\prime}$ correspond to the first $q_{n}$ and remaining $p_{n}-q$ components of $\boldsymbol{\beta}$, respectively. Let

$$
Q_{n}(\boldsymbol{\theta} \mid \boldsymbol{\beta})=-2 l(\boldsymbol{\theta})+\lambda_{n} \boldsymbol{\theta}^{\prime} D(\boldsymbol{\beta}) \boldsymbol{\theta},
$$

where $D(\boldsymbol{\beta})=\operatorname{diag}\left(\beta_{1}^{-2}, \beta_{2}^{-2}, \ldots, \beta_{q_{n}}^{-2}, \beta_{q_{n}+1}^{-2}, \ldots, \beta_{p_{n}}^{-2}\right)$ and $l(\boldsymbol{\theta})$ is the $p_{n}$-dimensional $\log$ partial likelihood of the reduced model. Let $\dot{Q}(\boldsymbol{\theta} \mid \boldsymbol{\beta})$ and $\ddot{Q}(\boldsymbol{\theta} \mid \boldsymbol{\beta})$ be the first and second derivatives of $Q(\boldsymbol{\theta} \mid \boldsymbol{\beta})$ with respective to $\boldsymbol{\theta}$, respectively. Then

$$
\begin{gathered}
\dot{Q}(\boldsymbol{\theta} \mid \boldsymbol{\beta})=-2 \dot{l}(\boldsymbol{\theta})+2 \lambda_{n} D(\boldsymbol{\beta}) \boldsymbol{\theta}, \\
\ddot{Q}(\boldsymbol{\theta} \mid \boldsymbol{\beta})=-2 \ddot{l}(\boldsymbol{\theta})+2 \lambda_{n} D(\boldsymbol{\beta}) .
\end{gathered}
$$

Lemma 3 Let $M_{n}=\max \left\{2 / a_{n}, 2 b_{n}\right\}$. Define $\mathcal{H}_{n} \equiv\left\{\boldsymbol{\beta}=\left(\boldsymbol{\beta}_{1}^{\prime}, \boldsymbol{\beta}_{2}^{\prime}\right)^{\prime}:\left|\boldsymbol{\beta}_{1}\right|=\left(\left|\beta_{1}\right|, \ldots,\left|\beta_{q_{n}}\right|\right)^{\prime} \in\right.$ $\left.\left[1 / M_{n}, M_{n}\right]^{q_{n}}, 0<\left\|\boldsymbol{\beta}_{2}\right\|_{2} \leq \delta_{n} \sqrt{p_{n} / n},\right\}$, where $\delta_{n}$ is a sequence of positive real numbers satisfying $\delta_{n} \rightarrow \infty$ and $p_{n} \delta_{n}^{2} / \lambda_{n} \rightarrow 0$. For any given $\boldsymbol{\beta} \in \mathcal{H}_{n}$, define

$$
Q_{n}(\boldsymbol{\theta} \mid \boldsymbol{\beta})=-2 l(\boldsymbol{\theta})+\lambda_{n} \boldsymbol{\theta}^{\prime} D(\boldsymbol{\beta}) \boldsymbol{\theta},
$$


where $l(\boldsymbol{\theta})$ is the $p_{n}$-dimensional log-partial likelihood and $D(\boldsymbol{\beta})=\operatorname{diag}\left(\beta_{1}^{-2}, \ldots, \beta_{p_{n}}^{-2}\right)$. Let $g(\boldsymbol{\beta})=\left(g_{1}(\boldsymbol{\beta})^{\prime}, g_{2}(\boldsymbol{\beta})^{\prime}\right)^{\prime}$ be a solution to $\dot{Q}(\boldsymbol{\theta} \mid \boldsymbol{\beta})=\mathbf{0}$, where

$$
\dot{Q}(\boldsymbol{\theta} \mid \boldsymbol{\beta})=-2 \dot{l}(\boldsymbol{\theta})+2 \lambda_{n} D(\boldsymbol{\beta}) \boldsymbol{\theta},
$$

is the derivative of $Q(\boldsymbol{\theta} \mid \boldsymbol{\beta})$ with respective to $\boldsymbol{\theta}$. Assume that conditions (C1) - (C6) hold. Then, as $n \rightarrow \infty$, with probability tending to 1 ,

(a) $\sup _{\boldsymbol{\beta} \in \mathcal{H}_{n}} \frac{\left\|g_{2}(\boldsymbol{\beta})\right\|_{2}}{\left\|\boldsymbol{\beta}_{2}\right\|_{2}} \leq \frac{1}{K_{1}}$, for some constant $K_{1}>1$;

(b) $\left|g_{1}(\boldsymbol{\beta})\right| \in\left[1 / M_{n}, M_{n}\right]^{q_{n}}$.

Proof. By the first-order Taylor expansion and the definition of $g(\boldsymbol{\beta})$, we have

$$
\dot{Q}\left(\boldsymbol{\beta}_{0} \mid \boldsymbol{\beta}\right)=\dot{Q}\{g(\boldsymbol{\beta}) \mid \boldsymbol{\beta}\}+\ddot{Q}\left(\boldsymbol{\beta}^{*} \mid \boldsymbol{\beta}\right)\left\{\boldsymbol{\beta}_{0}-g(\boldsymbol{\beta})\right\}=\ddot{Q}\left(\boldsymbol{\beta}^{*} \mid \boldsymbol{\beta}\right)\left\{\boldsymbol{\beta}_{0}-g(\boldsymbol{\beta})\right\}
$$

where $\boldsymbol{\beta}_{0}$ is the true parameter vector, and $\boldsymbol{\beta}^{*}$ lies between $\boldsymbol{\beta}_{0}$ and $g(\boldsymbol{\beta})$. Rearranging terms, we have

$$
\ddot{Q}\left(\boldsymbol{\beta}^{*} \mid \boldsymbol{\beta}\right) g(\boldsymbol{\beta})=-\dot{Q}\left(\boldsymbol{\beta}_{0} \mid \boldsymbol{\beta}\right)+\ddot{Q}\left(\boldsymbol{\beta}^{*} \mid \boldsymbol{\beta}\right) \boldsymbol{\beta}_{0}
$$

which can be rewritten as

$$
\begin{aligned}
\left\{-2 \ddot{l}\left(\boldsymbol{\beta}^{*}\right)+2 \lambda_{n} D(\boldsymbol{\beta})\right\} g(\boldsymbol{\beta}) & =-\left\{-2 \dot{l}\left(\boldsymbol{\beta}_{0}\right)+2 \lambda_{n} D(\boldsymbol{\beta}) \boldsymbol{\beta}_{0}\right\}+\left\{-2 \ddot{l}\left(\boldsymbol{\beta}^{*}\right)+2 \lambda_{n} D(\boldsymbol{\beta})\right\} \boldsymbol{\beta}_{0} \\
& =2 i\left(\boldsymbol{\beta}_{0}\right)-2 \ddot{l}\left(\boldsymbol{\beta}^{*}\right) \boldsymbol{\beta}_{0} .
\end{aligned}
$$

Write $H(\boldsymbol{\beta}) \equiv-n^{-1} \ddot{l}(\boldsymbol{\beta})$, we have

$$
\left\{H\left(\boldsymbol{\beta}^{*}\right)+\frac{\lambda_{n}}{n} D(\boldsymbol{\beta})\right\} g(\boldsymbol{\beta})=H\left(\boldsymbol{\beta}^{*}\right) \boldsymbol{\beta}_{0}+\frac{1}{n} i\left(\boldsymbol{\beta}_{0}\right),
$$

which can be further written as

$$
\left\{g(\boldsymbol{\beta})-\boldsymbol{\beta}_{0}\right\}+\frac{\lambda_{n}}{n} H\left(\boldsymbol{\beta}^{*}\right)^{-1} D(\boldsymbol{\beta}) g(\boldsymbol{\beta})=\frac{1}{n} H\left(\boldsymbol{\beta}^{*}\right)^{-1} i\left(\boldsymbol{\beta}_{0}\right) .
$$


Now we partition $H\left(\boldsymbol{\beta}^{*}\right)^{-1}$ into

$$
H\left(\boldsymbol{\beta}^{*}\right)^{-1}=\left[\begin{array}{cc}
A & B \\
B^{\prime} & G
\end{array}\right]
$$

and partition $D(\boldsymbol{\beta})$ into

$$
D(\boldsymbol{\beta})=\left[\begin{array}{ll}
D_{1}\left(\boldsymbol{\beta}_{1}\right) & \mathbf{0} \\
\mathbf{0}^{\prime} & D_{2}\left(\boldsymbol{\beta}_{2}\right)
\end{array}\right]
$$

where $D_{1}\left(\boldsymbol{\beta}_{1}\right)=\operatorname{diag}\left(\left|\beta_{1}\right|^{-2}, \ldots,\left|\beta_{q_{n}}\right|^{-2}\right)$ and $D_{2}\left(\boldsymbol{\beta}_{2}\right)=\operatorname{diag}\left(\left|\beta_{q_{n}+1}\right|^{-2}, \ldots,\left|\beta_{p_{n}}\right|^{-2}\right)$. Then (28) can be re-written as

$$
\left(\begin{array}{c}
g_{1}(\boldsymbol{\beta})-\boldsymbol{\beta}_{01} \\
g_{2}(\boldsymbol{\beta})
\end{array}\right)+\frac{\lambda_{n}}{n}\left(\begin{array}{c}
A D_{1}\left(\boldsymbol{\beta}_{1}\right) g_{1}(\boldsymbol{\beta})+B D_{2}\left(\boldsymbol{\beta}_{2}\right) g_{2}(\boldsymbol{\beta}) \\
B^{\prime} D_{1}\left(\boldsymbol{\beta}_{1}\right) g_{1}(\boldsymbol{\beta})+G D_{2}\left(\boldsymbol{\beta}_{2}\right) g_{2}(\boldsymbol{\beta})
\end{array}\right)=\frac{1}{n} H\left(\boldsymbol{\beta}^{*}\right)^{-1} i\left(\boldsymbol{\beta}_{0}\right) .
$$

Moreover, it follows from (13), (14), and condition (C5) that

$$
\left\|n^{-1} H\left(\boldsymbol{\beta}^{*}\right)^{-1} i\left(\boldsymbol{\beta}_{0}\right)\right\|_{2}=O_{p}\left(\sqrt{p_{n} / n}\right) .
$$

Therefore,

$$
\sup _{\boldsymbol{\beta} \in \mathcal{H}_{n}}\left\|g_{2}(\boldsymbol{\beta})+\frac{\lambda_{n}}{n} B^{\prime} D_{1}\left(\boldsymbol{\beta}_{1}\right) g_{1}(\boldsymbol{\beta})+\frac{\lambda_{n}}{n} G D_{2}\left(\boldsymbol{\beta}_{2}\right) g_{2}(\boldsymbol{\beta})\right\|_{2}=O_{p}\left(\sqrt{p_{n} / n}\right) .
$$

Furthermore,

$$
\begin{aligned}
\left\|g(\boldsymbol{\beta})-\boldsymbol{\beta}_{0}\right\|_{2} & =\left\|-\left\{H\left(\boldsymbol{\beta}^{*}\right)+\frac{\lambda_{n}}{n} D(\boldsymbol{\beta})\right\}^{-1}\left\{\frac{\lambda_{n}}{n} D(\boldsymbol{\beta}) \boldsymbol{\beta}_{0}-\frac{1}{n} i\left(\boldsymbol{\beta}_{0}\right)\right\}\right\|_{2} \\
& \leq\left\|\left\{H\left(\boldsymbol{\beta}^{*}\right)\right\}^{-1}\left\{\frac{\lambda_{n}}{n} D(\boldsymbol{\beta}) \boldsymbol{\beta}_{0}-\frac{1}{n} i\left(\boldsymbol{\beta}_{0}\right)\right\}\right\|_{2} \\
& \leq\left\|\left\{H\left(\boldsymbol{\beta}^{*}\right)\right\}^{-1}\right\|_{2} \cdot\left\{\left\|\frac{\lambda_{n}}{n} D_{1}\left(\boldsymbol{\beta}_{1}\right) \boldsymbol{\beta}_{01}\right\|_{2}+\left\|\frac{1}{n} i\left(\boldsymbol{\beta}_{0}\right)\right\|_{2}\right\} \\
& =O_{p}(1)\left\{O\left(n^{-1} \lambda_{n} M_{n}^{3} \sqrt{q_{n}}\right)+O_{p}\left(\sqrt{p_{n} / n}\right)\right\} \\
& =O_{p}\left(\sqrt{p_{n} / n}\right),
\end{aligned}
$$


where the first equality follows from (27) and the fourth step follows from (14), condition (C3), $\left\|n^{-1} \lambda_{n} D_{1}\left(\boldsymbol{\beta}_{1}\right) \boldsymbol{\beta}_{01}\right\|_{2}=O\left(n^{-1} \lambda_{n} M_{n}^{3} \sqrt{q_{n}}\right)$, and $\left\|n^{-1} i\left(\boldsymbol{\beta}_{0}\right)\right\|_{2}=O_{p}\left(\sqrt{p_{n} / n}\right)$, and the last step holds since $n^{-1} \lambda_{n} M_{n}^{3} \sqrt{q_{n}}=o(1 / \sqrt{n})$ under condition (C6). Hence,

$$
\|g(\boldsymbol{\beta})\|_{2} \leq\left\|\boldsymbol{\beta}_{0}\right\|_{2}+\left\|g(\boldsymbol{\beta})-\boldsymbol{\beta}_{0}\right\|_{2}=O_{p}\left(M_{n} \sqrt{q_{n}}\right)
$$

Also note that $\|B\|_{2}=O_{p}(1)$ since $\left\|B B^{\prime}\right\|_{2} \leq\left\|A^{2}+B B^{\prime}\right\|_{2}+\left\|A^{2}\right\|_{2} \leq 2\left\|A^{2}+B B^{\prime}\right\|_{2} \leq$ $2\left\|H\left(\boldsymbol{\beta}^{*}\right)^{-2}\right\|_{2}=O_{p}(1)$. This, combined with 32 , implies that

$$
\sup _{\boldsymbol{\beta} \in \mathcal{H}_{n}}\left\|\frac{\lambda_{n}}{n} B^{\prime} D_{1}\left(\boldsymbol{\beta}_{1}\right) g_{1}(\boldsymbol{\beta})\right\|_{2} \leq \frac{\lambda_{n}}{n} \sup _{\boldsymbol{\beta} \in \mathcal{H}_{n}}\|B\|_{2}\left\|D_{1}\left(\boldsymbol{\beta}_{1}\right)\right\|_{2}\left\|g_{1}(\boldsymbol{\beta})\right\|_{2}=O_{p}\left(\frac{\lambda_{n} M_{n}^{3} \sqrt{q_{n}}}{n}\right)=o(1 / \sqrt{n}) .
$$

It then follows that

$$
\sup _{\boldsymbol{\beta} \in \mathcal{H}_{n}}\left\|g_{2}(\boldsymbol{\beta})+\frac{\lambda_{n}}{n} G D_{2}\left(\boldsymbol{\beta}_{2}\right) g_{2}(\boldsymbol{\beta})\right\|_{2} \leq O_{p}\left(\sqrt{p_{n} / n}\right)+o(1 / \sqrt{n})=O_{p}\left(\sqrt{p_{n} / n}\right) .
$$

Since $G$ is positive definite and symmetric with probability tending to one, by the spectral decomposition theorem, $G=\sum_{i=1}^{p_{n}-q_{n}} r_{2 i} \mathbf{u}_{2 i} \mathbf{u}_{2 i}^{\prime}$, where $r_{2 i}$ and $\mathbf{u}_{2 i}$ are the eigenvalues and eigenvectors of $G$, respectively. Now with probability tending to one,

$$
\begin{aligned}
\frac{\lambda_{n}}{n}\left\|G D_{2}\left(\boldsymbol{\beta}_{2}\right) g_{2}(\boldsymbol{\beta})\right\|_{2} & =\frac{\lambda_{n}}{n}\left\|\left(\sum_{i=1}^{p_{n}-q_{n}} r_{2 i} \mathbf{u}_{2 i} \mathbf{u}_{2 i}^{\prime}\right) D_{2}\left(\boldsymbol{\beta}_{2}\right) g_{2}(\boldsymbol{\beta})\right\|_{2} \\
& \geq \frac{\lambda_{n}}{n}\left\|C_{2}\left(\sum_{i=1}^{p_{n}-q_{n}} \mathbf{u}_{2 i} \mathbf{u}_{2 i}^{\prime}\right) D_{2}\left(\boldsymbol{\beta}_{2}\right) g_{2}(\boldsymbol{\beta})\right\|_{2} \\
& \geq C_{2}\left\|\frac{\lambda_{n}}{n} D_{2}\left(\boldsymbol{\beta}_{2}\right) g_{2}(\boldsymbol{\beta})\right\|_{2},
\end{aligned}
$$

where the first inequality is due to (14) and condition (C4) since we can assume that for all $i=1, \ldots, p-q, r_{2 i} \in\left(C_{2}, C_{3}\right)$ for some $C_{2}<C_{3}<\infty$ with probability tending to one. Therefore with probability tending to one,

$$
C_{2}\left\|\frac{\lambda_{n}}{n} D_{2}\left(\boldsymbol{\beta}_{2}\right) g_{2}(\boldsymbol{\beta})\right\|_{2}-\left\|g_{2}(\boldsymbol{\beta})\right\|_{2} \leq\left\|g_{2}(\boldsymbol{\beta})+\frac{\lambda_{n}}{n} G D_{2}\left(\boldsymbol{\beta}_{2}\right) g_{2}(\boldsymbol{\beta})\right\|_{2} \leq \delta_{n} \sqrt{p_{n} / n}
$$


where $\delta_{n}$ diverges to $\infty$. Let $\mathbf{m}_{g_{2}(\boldsymbol{\beta}) / \boldsymbol{\beta}_{2}}=\left(g_{2}\left(\beta_{q_{n}+1}\right) / \beta_{q_{n}+1}, \ldots, g_{2}\left(\beta_{p_{n}}\right) / \beta_{p_{n}}\right)^{\prime}$. Because $\left\|\boldsymbol{\beta}_{2}\right\|_{2} \leq \delta_{n} \sqrt{p_{n} / n}$, we have

$$
C_{2}\left\|\frac{\lambda_{n}}{n} D_{2}\left(\boldsymbol{\beta}_{2}\right) g_{2}(\boldsymbol{\beta})\right\|_{2}=C_{2} \frac{\lambda_{n}}{n}\left\|D_{2}\left(\boldsymbol{\beta}_{2}\right)^{1 / 2} \mathbf{m}_{g_{2}(\boldsymbol{\beta}) / \boldsymbol{\beta}_{2}}\right\|_{2} \geq C_{2} \frac{\lambda_{n}}{n} \frac{\sqrt{n}}{\delta_{n} \sqrt{p_{n}}}\left\|\mathbf{m}_{g_{2}(\boldsymbol{\beta}) / \boldsymbol{\beta}_{2}}\right\|_{2},
$$

and

$$
\left\|g_{2}(\boldsymbol{\beta})\right\|_{2}=\left\|D_{2}\left(\boldsymbol{\beta}_{2}\right)^{-1 / 2} \mathbf{m}_{g_{2}(\boldsymbol{\beta}) / \boldsymbol{\beta}_{2}}\right\|_{2} \leq\left\|D_{2}\left(\boldsymbol{\beta}_{2}\right)^{-1 / 2}\right\|_{2} \cdot\left\|\mathbf{m}_{g_{2}(\boldsymbol{\beta}) / \boldsymbol{\beta}_{2}}\right\|_{2} \leq \frac{\delta_{n} \sqrt{p_{n}}}{\sqrt{n}}\left\|\mathbf{m}_{g_{2}(\boldsymbol{\beta}) / \boldsymbol{\beta}_{2}}\right\|_{2} .
$$

Hence it follows from (35), (36), and (37) that with probability tending to one,

$$
C_{2} \frac{\lambda_{n}}{n} \frac{\sqrt{n}}{\delta_{n} \sqrt{p_{n}}}\left\|\mathbf{m}_{g_{2}(\boldsymbol{\beta}) / \boldsymbol{\beta}_{2}}\right\|_{2}-\frac{\delta_{n} \sqrt{p_{n}}}{\sqrt{n}}\left\|\mathbf{m}_{g_{2}(\boldsymbol{\beta}) / \boldsymbol{\beta}_{2}}\right\|_{2} \leq \delta_{n} \sqrt{p_{n} / n}
$$

This implies that with probability tending to one,

$$
\left\|\mathbf{m}_{g_{2}(\boldsymbol{\beta}) / \boldsymbol{\beta}_{2}}\right\|_{2} \leq \frac{1}{\lambda_{n} /\left(C_{1} p \delta_{n}^{2}\right)-1}<\frac{1}{K_{1}}
$$

for some constant $K_{1}>1$ provided that $\lambda_{n} /\left(p_{n} \delta_{n}^{2}\right) \rightarrow \infty$ as $n \rightarrow \infty$. Now from (38), we have

$$
\left\|g_{2}(\boldsymbol{\beta})\right\|_{2} \leq\left\|\mathbf{m}_{g_{2}(\boldsymbol{\beta}) / \boldsymbol{\beta}_{2}}\right\|_{2} \max _{q+1 \leq j \leq p}\left|\beta_{j}\right| \leq\left\|\mathbf{m}_{g_{2}(\boldsymbol{\beta}) / \boldsymbol{\beta}_{2}}\right\|_{2}\left\|\boldsymbol{\beta}_{2}\right\|_{2} \leq \frac{1}{K_{1}}\left\|\boldsymbol{\beta}_{2}\right\|_{2},
$$

with probability tending to one. Thus

$$
\operatorname{pr}\left(\sup _{\boldsymbol{\beta} \in \mathcal{H}_{n}} \frac{\left\|g_{2}(\boldsymbol{\beta})\right\|_{2}}{\left\|\boldsymbol{\beta}_{2}\right\|_{2}}<\frac{1}{K_{1}}\right) \rightarrow 1 \quad \text { as } n \rightarrow \infty
$$

and (a) is proved.

To prove part (b), we first note from 39 that as $n \rightarrow \infty, \operatorname{pr}\left(\left\|\mathbf{m}_{g_{2}(\boldsymbol{\beta}) / \boldsymbol{\beta}_{2}}\right\|_{2} \leq \delta_{n} \sqrt{p_{n} / n}\right) \rightarrow$ 1. Therefore it is sufficient to show that for any $\boldsymbol{\beta} \in \mathcal{H}_{n},\left|g_{1}(\boldsymbol{\beta})\right| \in\left[1 / M_{n}, M_{n}\right]^{q_{n}}$ with probability tending to 1 . By 29 and (30), we have

$$
\sup _{\boldsymbol{\beta} \in \mathcal{H}_{n}}\left\|\left(g_{1}(\boldsymbol{\beta})-\boldsymbol{\beta}_{01}\right)+\frac{\lambda_{n}}{n} A D_{1}\left(\boldsymbol{\beta}_{1}\right) g_{1}(\boldsymbol{\beta})+\frac{\lambda_{n}}{n} B D_{2}\left(\boldsymbol{\beta}_{2}\right) g_{2}(\boldsymbol{\beta})\right\|_{2}=O_{p}\left(\sqrt{p_{n} / n}\right) .
$$


Similar to (33), it can be shown that

$$
\sup _{\boldsymbol{\beta} \in \mathcal{H}_{n}}\left\|\frac{\lambda_{n}}{n} A D_{1}\left(\boldsymbol{\beta}_{1}\right) g_{1}(\boldsymbol{\beta})\right\|_{2}=O_{p}\left(\frac{\lambda_{n} M_{n}^{3} \sqrt{q_{n}}}{n}\right)=o_{p}(1 / \sqrt{n}),
$$

where the last equality holds trivially under condition (C6). Furthermore, with probability tending to one,

$$
\sup _{\boldsymbol{\beta} \in \mathcal{H}_{n}}\left\|\frac{\lambda_{n}}{n} B D_{2}\left(\boldsymbol{\beta}_{2}\right) g_{2}(\boldsymbol{\beta})\right\|_{2} \leq \frac{\lambda_{n}}{n} \sup _{\boldsymbol{\beta} \in \mathcal{H}_{n}}\|B\|_{2} \cdot\left\|D_{2}\left(\boldsymbol{\beta}_{2}\right) g_{2}(\boldsymbol{\beta})\right\|_{2} \leq \frac{\lambda_{n}}{n} \sqrt{2 K_{3}}\left(\delta_{n} \sqrt{\frac{p_{n}}{n}}\right)^{2},
$$

for some $K_{3}>0$, since $\left\|g_{2}(\boldsymbol{\beta})\right\| \leq \delta_{n} \sqrt{p_{n} / n},\|B\|_{2}=O_{p}(1)$ and $\left\|D_{2}\left(\boldsymbol{\beta}_{2}\right)\right\|_{2} \leq \delta_{n} \sqrt{p_{n} / n}$. Therefore, combing (40), (41) and (42) gives

$$
\sup _{\boldsymbol{\beta} \in \mathcal{H}_{n}}\left\|g_{1}(\boldsymbol{\beta})-\boldsymbol{\beta}_{01}\right\|_{2} \leq \frac{\lambda_{n}}{n} \sqrt{2 K_{3}}\left(\delta_{n} \sqrt{\frac{p_{n}}{n}}\right)^{2}+\frac{\delta_{n} \sqrt{p_{n}}}{\sqrt{n}},
$$

with probability tending to one. Because $\lambda_{n} / n \rightarrow 0$ and $\delta_{n} \sqrt{p_{n} / n}=\sqrt{p_{n} \delta_{n}^{2} / \lambda_{n}} \sqrt{\lambda_{n} / n} \rightarrow 0$ as $n \rightarrow \infty$, we have $\operatorname{pr}\left(\left|g_{1}(\boldsymbol{\beta})\right| \in\left[1 / M_{n}, M_{n}\right]^{q_{n}}\right) \rightarrow 1$. This completes the proof of part (b).

Lemma 4 Let $\boldsymbol{\beta}_{1}$ be the first $q_{n}$ components of $\boldsymbol{\beta}$. Define $f\left(\boldsymbol{\beta}_{1}\right)=\arg \min _{\boldsymbol{\theta}_{1}}\left\{Q_{n 1}\left(\boldsymbol{\theta}_{1} \mid \boldsymbol{\beta}_{1}\right)\right\}$, where $Q_{n 1}\left(\boldsymbol{\theta}_{1} \mid \boldsymbol{\beta}_{1}\right)=-2 l_{n 1}\left(\boldsymbol{\theta}_{1}\right)+\lambda_{n} \boldsymbol{\theta}_{1}^{\prime} D_{1}\left(\boldsymbol{\beta}_{1}\right) \boldsymbol{\theta}_{1}$, is a weighted $\ell_{2}$-penalized -2 log-pseudo likelihood for the oracle model of model size $q_{n}$, and $D_{1}\left(\boldsymbol{\beta}_{1}\right)=\operatorname{diag}\left(\beta_{1}^{-2}, \beta_{2}^{-2}, \ldots, \beta_{q_{n}}^{-2}\right)$. Assume that conditions (C1) - (C6) hold. Then with probability tending to one,

(a) $f\left(\boldsymbol{\beta}_{1}\right)$ is a contraction mapping from $\left[1 / M_{n}, M_{n}\right]^{q_{n}}$ to itself;

(b) $\sqrt{n} \mathbf{d}_{n}^{\prime} \Gamma_{11}^{-1 / 2} \Omega_{11}\left(\hat{\boldsymbol{\beta}}_{1}^{\circ}-\boldsymbol{\beta}_{01}\right) \rightarrow N(0,1)$, for any $q_{n}$-dimensional vector $\mathbf{d}_{n}$ such that $\mathbf{d}_{n}^{\prime} \mathbf{d}_{n}=1$ and where $\hat{\boldsymbol{\beta}}_{1}^{\circ}$ is the unique fixed point of $f\left(\boldsymbol{\beta}_{1}\right)$ and $\Sigma_{11}$ and $\Omega_{11}$ are the first $q_{n} \times q_{n}$ submatrices of $\Sigma$ and $\Omega$, respectively. 
Proof: (a) First we show that $f(\cdot)$ is a mapping from $\left[1 / M_{n}, M_{n}\right]^{q_{n}}$ to itself with probability tending to one. Again through a first order Taylor expansion, we have

$$
\left\{f\left(\boldsymbol{\beta}_{1}\right)-\boldsymbol{\beta}_{01}\right\}+\frac{\lambda_{n}}{n} H_{1}\left(\boldsymbol{\beta}_{1}^{*}\right)^{-1} D_{1}\left(\boldsymbol{\beta}_{1}\right) f\left(\boldsymbol{\beta}_{1}\right)=\frac{1}{n} H_{1}\left(\boldsymbol{\beta}_{1}^{*}\right)^{-1} i_{1}\left(\boldsymbol{\beta}_{01}\right),
$$

where $H_{1}\left(\boldsymbol{\beta}_{1}^{*}\right)=-n^{-1} \ddot{l}_{1}\left(\boldsymbol{\beta}_{1}^{*}\right)$ exists and is invertible for $\boldsymbol{\beta}_{1}^{*}$ between $\boldsymbol{\beta}_{01}$ and $f\left(\boldsymbol{\beta}_{1}\right)$. We have

$$
\sup _{\left|\boldsymbol{\beta}_{1}\right| \in\left[1 / M_{n}, M_{n}\right]^{q n}}\left\|f\left(\boldsymbol{\beta}_{1}\right)-\boldsymbol{\beta}_{01}+\frac{\lambda_{n}}{n} H_{1}\left(\boldsymbol{\beta}_{1}^{*}\right)^{-1} D_{1}\left(\boldsymbol{\beta}_{1}\right) f\left(\boldsymbol{\beta}_{1}\right)\right\|_{2}=O_{p}\left(\sqrt{q_{n} / n}\right),
$$

where the right-hand side follows in the same fashion as 33 . Similar to (33) we have

$$
\sup _{\left|\boldsymbol{\beta}_{1}\right| \in\left[1 / M_{0}, M_{0}\right]^{q_{n}}}\left\|\frac{\lambda_{n}}{n} H_{1}\left(\boldsymbol{\beta}_{1}^{*}\right)^{-1} D_{1}\left(\boldsymbol{\beta}_{1}\right) f\left(\boldsymbol{\beta}_{1}\right)\right\|_{2}=O_{p}\left(\frac{\lambda_{n} M_{n}^{3}}{\sqrt{n}} \sqrt{\frac{q_{n}}{n}}\right)=o_{p}(1 / \sqrt{n}) .
$$

Therefore, with probability tending to one

$$
\sup _{\left|\boldsymbol{\beta}_{1}\right| \in\left[1 / M_{n}, M_{n}\right]^{q_{n}}}\left\|f\left(\boldsymbol{\beta}_{1}\right)-\boldsymbol{\beta}_{01}\right\|_{2} \leq \delta_{n} \sqrt{q_{n} / n}
$$

where $\delta_{n}$ is a sequence of real numbers diverging to $\infty$ and satisfies $\delta_{n} \sqrt{p_{n} / n} \rightarrow 0$. As a result, we have

$$
\operatorname{pr}\left(f\left(\boldsymbol{\beta}_{1}\right) \in\left[1 / M_{n}, M_{n}\right]^{q_{n}}\right) \rightarrow 1
$$

as $n \rightarrow \infty$. Hence $f(\cdot)$ is a mapping from the region $\left[1 / M_{n}, M_{n}\right]^{q_{n}}$ to itself. To prove that $f(\cdot)$ is a contraction mapping, we need to further show that

$$
\sup _{\left|\boldsymbol{\beta}_{1}\right| \in\left[1 / M_{n}, M_{n}\right]^{q_{n}}}\left\|\dot{f}\left(\boldsymbol{\beta}_{1}\right)\right\|_{2}=o_{p}(1) .
$$

Since $f\left(\boldsymbol{\beta}_{1}\right)$ is a solution to $\dot{Q}_{1}\left(\boldsymbol{\theta}_{1} \mid \boldsymbol{\beta}_{1}\right)=0$, we have

$$
-\frac{1}{n} \dot{l}_{1}\left(f\left(\boldsymbol{\beta}_{1}\right)\right)=-\frac{\lambda_{n}}{n} D_{1}\left(\boldsymbol{\beta}_{1}\right) f\left(\boldsymbol{\beta}_{1}\right) .
$$


Taking the derivative of 460 with respect to $\boldsymbol{\beta}_{1}^{\prime}$ and rearranging terms, we obtain

$$
\left\{H_{1}\left(f\left(\boldsymbol{\beta}_{1}\right)\right)+\frac{\lambda_{n}}{n} D_{1}\left(\boldsymbol{\beta}_{1}\right)\right\} \dot{f}\left(\boldsymbol{\beta}_{1}\right)=\frac{2 \lambda_{n}}{n} \operatorname{diag}\left\{f_{1}\left(\boldsymbol{\beta}_{1}\right) / \beta_{1}^{3}, \ldots, f_{q_{n}}\left(\boldsymbol{\beta}_{1}\right) / \beta_{q_{n}}^{3}\right\} .
$$

With probability tending to one, we have

$$
\sup _{\left|\boldsymbol{\beta}_{1}\right| \in\left[1 / M_{n}, M_{n}\right]^{q_{n}}} \frac{2 \lambda_{n}}{n}\left\|\operatorname{diag}\left\{f_{1}\left(\boldsymbol{\beta}_{1}\right) / \beta_{1}^{3}, \ldots, f_{q_{n}}\left(\boldsymbol{\beta}_{1}\right) / \beta_{q_{n}}^{3}\right\}\right\|_{2}=O_{p}\left(\frac{\lambda_{n} M_{n}^{4}}{n}\right)=o_{p}(1),
$$

where the last step follows from condition (C6). This, combined with 47) implies that

$$
\sup _{\left|\boldsymbol{\beta}_{1}\right| \in\left[1 / M_{n}, M_{n}\right]^{q_{n}}}\left\|\left\{H_{1}\left(f\left(\boldsymbol{\beta}_{1}\right)\right)+\frac{\lambda_{n}}{n} D_{1}\left(\boldsymbol{\beta}_{1}\right)\right\} \dot{f}\left(\boldsymbol{\beta}_{1}\right)\right\|_{2}=o_{p}(1) .
$$

Now, it can be shown that probability tending to one,

$$
\left\|H_{1}\left(f\left(\boldsymbol{\beta}_{1}\right)\right) \dot{f}\left(\boldsymbol{\beta}_{1}\right)\right\|_{2} \geq\left\|\dot{f}\left(\boldsymbol{\beta}_{1}\right)\right\|_{2} \cdot\left\|H_{1}\left(f\left(\boldsymbol{\beta}_{1}\right)\right)^{-1}\right\|_{2}^{-1} \geq \frac{1}{K_{2}}\left\|\dot{f}\left(\boldsymbol{\beta}_{1}\right)\right\|_{2},
$$

for some $K_{2}>0$, and that

$$
\frac{\lambda_{n}}{n}\left\|D_{1}\left(\boldsymbol{\beta}_{1}\right) \dot{f}\left(\boldsymbol{\beta}_{1}\right)\right\|_{2} \geq \frac{\lambda_{n}}{n}\left\|\dot{f}\left(\boldsymbol{\beta}_{1}\right)\right\|_{2}\left\|D_{1}\left(\boldsymbol{\beta}_{1}\right)^{-1}\right\|_{2}^{-1} \geq \frac{\lambda_{n}}{n} \frac{1}{M_{n}^{2}}\left\|\dot{f}\left(\boldsymbol{\beta}_{1}\right)\right\|_{2} .
$$

Therefore, combining the above two inequalities with (47) and (48) gives

$$
\left(\frac{1}{K_{2}}-\frac{\lambda_{n}}{n M_{n}^{2}}\right) \sup _{\left|\boldsymbol{\beta}_{1}\right| \in\left[1 / M_{n}, M_{n}\right]^{q_{n}}}\left\|\dot{f}\left(\boldsymbol{\beta}_{1}\right)\right\|_{2}=o_{p}(1) .
$$

This, together with the fact that $\frac{\lambda_{n}}{n} \frac{1}{M_{n}^{2}}=o(1)$, implies that 45 holds. Therefore, with probability tending to one $f(\cdot)$ is a contraction mapping and consequently has a unique fixed point, say $\hat{\boldsymbol{\beta}}_{1}^{\circ}$, such that $\hat{\boldsymbol{\beta}}_{1}^{\circ}=f\left(\hat{\boldsymbol{\beta}}_{1}^{\circ}\right)$.

We next prove part (b). By (43) we have

$$
f\left(\boldsymbol{\beta}_{1}\right)=\left\{H_{1}\left(\boldsymbol{\beta}_{1}^{*}\right)+\frac{\lambda_{n}}{n} D_{1}\left(\boldsymbol{\beta}_{1}\right)\right\}^{-1}\left\{H_{1}\left(\boldsymbol{\beta}_{1}^{*}\right) \boldsymbol{\beta}_{01}+\frac{1}{n} i_{1}\left(\boldsymbol{\beta}_{01}\right)\right\} .
$$


Now,

$$
\begin{aligned}
\sqrt{n} \mathbf{d}_{n}^{\prime} \Gamma_{11}^{-1 / 2} \Omega_{11}\left(\hat{\boldsymbol{\beta}}_{1}^{\circ}-\boldsymbol{\beta}_{01}\right) & =\sqrt{n} \mathbf{d}_{n}^{\prime} \Gamma_{11}^{-1 / 2} \Omega_{11}\left[\left\{H_{1}\left(\boldsymbol{\beta}_{1}^{*}\right)+\frac{\lambda_{n}}{n} D_{1}\left(\hat{\boldsymbol{\beta}}_{1}^{\circ}\right)\right\}^{-1} H_{1}\left(\boldsymbol{\beta}_{1}^{*}\right)-I_{q_{n}}\right] \boldsymbol{\beta}_{01} \\
& +\sqrt{n} \mathbf{d}_{n}^{\prime} \Gamma_{11}^{-1 / 2} \Omega_{11}\left[\left\{H_{1}\left(\boldsymbol{\beta}_{1}^{*}\right)+\frac{\lambda_{n}}{n} D_{1}\left(\hat{\boldsymbol{\beta}}_{1}^{\circ}\right)\right\}^{-1} \frac{1}{n} i_{1}\left(\boldsymbol{\beta}_{01}\right)\right] \\
& =I_{1}+I_{2} .
\end{aligned}
$$

Note that for any two conformable invertible matrices $\Phi$ and $\Psi$, we have

$$
(\Phi+\Psi)^{-1}=\Phi^{-1}-\Phi^{-1} \Psi(\Phi+\Psi)^{-1},
$$

Thus we can rewrite $I_{1}$ as

$$
\begin{aligned}
I_{1} & =\sqrt{n} \mathbf{d}_{n}^{\prime} \Gamma_{11}^{-1 / 2} \Omega_{11}\left[\left\{H_{1}\left(\boldsymbol{\beta}_{1}^{*}\right)+\frac{\lambda_{n}}{n} D_{1}\left(\hat{\boldsymbol{\beta}}_{1}^{\circ}\right)\right\}^{-1} H_{1}\left(\boldsymbol{\beta}_{1}^{*}\right)-I_{q_{n}}\right] \boldsymbol{\beta}_{01} \\
& =-\frac{\lambda_{n}}{\sqrt{n}} \mathbf{d}_{n}^{\prime} \Gamma_{11}^{-1 / 2} \Omega_{11} H_{1}\left(\boldsymbol{\beta}_{1}^{*}\right)^{-1} D_{1}\left(\hat{\boldsymbol{\beta}}_{1}^{\circ}\right)\left\{H_{1}\left(\boldsymbol{\beta}_{1}^{*}\right)+\frac{\lambda_{n}}{n} D_{1}\left(\hat{\boldsymbol{\beta}}_{1}^{\circ}\right)\right\}^{-1} H_{1}\left(\boldsymbol{\beta}_{1}^{*}\right) \boldsymbol{\beta}_{01} .
\end{aligned}
$$

Moreoever

$$
\begin{aligned}
\left\|I_{1}\right\|_{2} & \leq \frac{\lambda_{n}}{\sqrt{n}}\left\|\Gamma_{11}^{-1 / 2} \Omega_{11}\right\|_{2}\left\|H_{1}\left(\boldsymbol{\beta}_{1}^{*}\right)^{-1}\right\|_{2}\left\|D_{1}\left(\hat{\boldsymbol{\beta}}_{1}^{\circ}\right)\right\|_{2}\left\|\left\{H_{1}\left(\boldsymbol{\beta}_{1}^{*}\right)+\frac{\lambda_{n}}{n} D_{1}\left(\hat{\boldsymbol{\beta}}_{1}^{\circ}\right)\right\}^{-1}\right\|_{2}\left\|H_{1}\left(\boldsymbol{\beta}_{1}^{*}\right)\right\|_{2}\left\|\boldsymbol{\beta}_{01}\right\|_{2} \\
& =\frac{\lambda_{n}}{\sqrt{n}} \cdot O(1) \cdot O_{p}(1) \cdot M_{n}^{2} \cdot O_{p}(1) \cdot O_{p}(1) \cdot M_{n} \sqrt{q_{n}} \\
& =O_{p}\left(\lambda_{n} M_{n}^{3} \sqrt{q_{n}} / \sqrt{n}\right)=o_{p}(1)
\end{aligned}
$$

where the first equality follows from $(14)$ and condition (C4), and the last equality is a 
consequence of condition (C6). Similarly, we can rewrite $I_{2}$ as

$$
\begin{aligned}
I_{2} & =\sqrt{n} \mathbf{d}_{n}^{\prime} \Gamma_{11}^{-1 / 2} \Omega_{11}\left[\left\{H_{1}\left(\boldsymbol{\beta}_{1}^{*}\right)+\frac{\lambda_{n}}{n} D_{1}\left(\hat{\boldsymbol{\beta}}_{1}^{\circ}\right)\right\}^{-1} \frac{1}{n} \dot{l}_{1}\left(\boldsymbol{\beta}_{01}\right)\right] \\
& =\mathbf{d}_{n}^{\prime} \Gamma_{11}^{-1 / 2} \Omega_{11} H_{1}\left(\boldsymbol{\beta}_{1}^{*}\right)^{-1} \frac{1}{\sqrt{n}} \dot{l}_{1}\left(\boldsymbol{\beta}_{01}\right) \\
& -\frac{\lambda_{n}}{\sqrt{n}} \mathbf{d}_{n}^{\prime} \Gamma_{11}^{-1 / 2} \Omega_{11} H_{1}\left(\boldsymbol{\beta}_{1}^{*}\right)^{-1} D_{1}\left(\hat{\boldsymbol{\beta}}_{1}^{\circ}\right)\left\{H_{1}\left(\boldsymbol{\beta}_{1}^{*}\right)^{-1}+\frac{\lambda_{n}}{n} D_{1}\left(\hat{\boldsymbol{\beta}}_{1}^{\circ}\right)\right\}^{-1} \frac{1}{n} \dot{l}_{1}\left(\boldsymbol{\beta}_{01}\right) \\
& =\mathbf{d}_{n}^{\prime} \Gamma_{11}^{-1 / 2} \Omega_{11} H_{1}\left(\boldsymbol{\beta}_{1}^{*}\right)^{-1} \frac{1}{\sqrt{n}} i_{n 1}\left(\boldsymbol{\beta}_{01}\right)+o_{p}(1) .
\end{aligned}
$$

We now establish the asymptotic normality of $n^{-1 / 2} \mathbf{d}_{n}^{\prime} \Gamma_{11}^{-1 / 2} \Omega_{11} H_{1}\left(\boldsymbol{\beta}_{1}^{*}\right)^{-1} i_{1}\left(\boldsymbol{\beta}_{01}\right)$ which will be derived in a similar manner to the proof of Theorem 2 in (?). By (14), (44), and the continuity of $\Omega$, we can deduce that $H_{1}\left(\boldsymbol{\beta}^{*}\right)=\Omega_{11}+o_{p}(1)$. This implies that

$$
\begin{aligned}
I_{2} & =n^{-1 / 2} \sum_{i=1}^{n} \mathbf{d}_{n}^{\prime} \Gamma_{11}^{-1 / 2} \Omega_{11} H_{1}\left(\boldsymbol{\beta}_{1}^{*}\right)^{-1} \mathbf{U}_{i 1}+o_{p}(1) \\
& =n^{-1 / 2} \sum_{i=1}^{n} \mathbf{d}_{n}^{\prime} \Gamma_{11}^{-1 / 2} \mathbf{U}_{i 1}+\left\{n^{-1 / 2} \sum_{i=1}^{n} \mathbf{d}_{n}^{\prime} \Gamma_{11}^{-1 / 2} \Omega_{11} \mathbf{U}_{i 1}\right\} o_{p}(1)+o_{p}(1) \\
& =I_{21}+I_{22} \cdot o_{p}(1)+o_{p}(1),
\end{aligned}
$$

where $\mathbf{U}_{i 1}$ consists of the first $q_{n}$ components of $\mathbf{U}_{i}$. Letting $Y_{n i}=n^{-1 / 2} \mathbf{d}_{n}^{\prime} \Gamma_{11}^{-1 / 2} \mathbf{U}_{i 1}$, then by condition (C5)

$$
\begin{aligned}
s_{n}^{2}=\sum_{i=1}^{n} \operatorname{var}\left(Y_{n i}\right) & =\frac{1}{n} \sum_{i=1}^{n} \mathbf{d}_{n}^{\prime} \Gamma_{11}^{-1 / 2} \operatorname{var}\left(\mathbf{U}_{i 1}\right) \Gamma_{11}^{-1 / 2} \mathbf{d}_{n} \\
& =\mathbf{d}_{n}^{\prime} \Gamma_{11}^{-1 / 2}\left\{\frac{1}{n} \sum_{i=1}^{n} \operatorname{var}\left(\mathbf{U}_{i 1}\right)\right\} \Gamma_{11}^{-1 / 2} \mathbf{d}_{n} \rightarrow 1 .
\end{aligned}
$$

To prove the asymptotic normality of $I_{21}$, we need to verify the Lindeberg condition: for all $\epsilon>0$,

$$
\frac{1}{s_{n}^{2}} \sum_{i=1}^{n} E\left\{Y_{n i}^{2} I\left(\left|Y_{n i}\right| \geq \epsilon s_{n}\right)\right\} \rightarrow 0
$$


as $n \rightarrow \infty$. Note that

$$
\begin{aligned}
\sum_{i=1}^{n} E\left(Y_{n i}^{4}\right) & =n^{-2} \sum_{i=1}^{n} E\left[\left\{\mathbf{d}_{n}^{\prime} \Gamma_{11}^{-1 / 2} \mathbf{U}_{i 1}\right\}^{4}\right] \\
& \leq n^{-2} \sum_{i=1}^{n} E\left[\left\|\mathbf{d}_{n}\right\|_{2}^{4} \cdot\left\|\Gamma_{11}^{-1 / 2}\right\|_{2}^{4} \cdot\left\|\mathbf{U}_{i 1}\right\|_{2}^{4}\right] \\
& =n^{-2} \operatorname{eigen}_{\max }^{2}\left\{\Gamma_{11}^{-1}\right\} \sum_{i=1}^{n} E\left(\left\|\mathbf{U}_{i 1}\right\|_{2}^{4}\right) \\
& =n^{-2} \text { eigen }_{\max }^{2}\left\{\Gamma_{11}^{-1}\right\} \sum_{i=1}^{n} \sum_{j=1}^{p_{n}} \sum_{k=1}^{p_{n}} E\left(U_{i j}^{2} U_{i k}^{2}\right) \\
& =O\left(p^{2} / n\right)
\end{aligned}
$$

where the first inequality is due to Cauchy-Schwarz, the second equality is due to $\left\|\mathbf{d}_{n}\right\|_{2}=1$ and the last step follows from conditions (C4) and (C5). Therefore for any $\epsilon>0$,

$$
\begin{aligned}
\frac{1}{s_{n}^{2}} \sum_{i=1}^{n} E\left\{Y_{n i}^{2} I\left(\left|Y_{n i}\right|>\epsilon s_{n}\right)\right\} & \leq \frac{1}{s_{n}^{2}} \sum_{i=1}^{n}\left\{E\left(Y_{n i}^{4}\right)\right\}^{1 / 2}\left[E\left\{I\left(\left|Y_{n i}\right|>\epsilon s_{n}\right)\right\}^{2}\right]^{1 / 2} \\
& \leq \frac{1}{s_{n}^{2}}\left\{\sum_{i=1}^{n} E\left(Y_{n i}^{4}\right)\right\}^{1 / 2} \cdot\left\{\sum_{i=1}^{n} \operatorname{pr}\left(\left|Y_{n i}\right|>\epsilon s_{n}\right)\right\}^{1 / 2} \\
& \leq \frac{1}{s_{n}^{2}}\left\{\sum_{i=1}^{n} E\left(Y_{n i}^{4}\right)\right\}^{1 / 2} \cdot\left\{\sum_{i=1}^{n} \frac{\operatorname{var}\left(Y_{n i}\right)}{\epsilon^{2} s_{n}^{2}}\right\}^{1 / 2} \\
& =\frac{1}{s_{n}^{2}}\left\{O\left(p^{2} / n\right)\right\}^{1 / 2} \frac{1}{\epsilon} \rightarrow 0 .
\end{aligned}
$$

Thus, (53) is satisfied and by the Lindeberg-Feller central limit theorem and Slutsky's theorem

$$
I_{21}=s_{n}\left(\frac{1}{s_{n}} \sum_{i=1}^{n} Y_{n i}\right) \rightarrow N(0,1)
$$


Similarly one can show that $I_{22}=O_{p}(1)$ and by Slutsky's theorem,

$$
\begin{aligned}
n^{-1 / 2} \mathbf{d}_{n}^{\prime} \Gamma_{11}^{-1 / 2} \Omega_{11} H_{1}\left(\boldsymbol{\beta}_{1}^{*}\right)^{-1} \dot{l}_{1}\left(\boldsymbol{\beta}_{01}\right) & =n^{-1 / 2} \sum_{i=1}^{n} \mathbf{d}_{n}^{\prime} \Gamma_{11}^{-1 / 2} \mathbf{U}_{i 1} \\
& +\left\{n^{-1 / 2} \sum_{i=1}^{n} \mathbf{d}_{n}^{\prime} \Gamma_{11}^{-1 / 2} \Omega_{11} \mathbf{U}_{i 1}\right\} o_{p}(1)+o_{p}(1) \\
& =I_{21}+I_{22} \cdot o_{p}(1)+o_{p}(1) \\
& \rightarrow N(0,1) .
\end{aligned}
$$

Hence, combining (49), (50), (52), and (55) gives

$$
\sqrt{n} \mathbf{d}_{n}^{\prime} \Gamma_{11}^{-1 / 2} \Omega_{11}\left(\hat{\boldsymbol{\beta}}_{1}^{\circ}-\boldsymbol{\beta}_{01}\right) \rightarrow N(0,1)
$$

which proves part (b).

\section{A.3 Proof of Theorem S1}

Part (a) of the theorem follows immediately from part (a) of Lemma S3. Part (b) of the theorem will follow from part (b) Lemma S4 and the following

$$
\operatorname{Pr}\left(\lim _{k \rightarrow \infty}\left\|g_{1}\left(\boldsymbol{\beta}^{(k)}\right)-\hat{\boldsymbol{\beta}}_{1}^{\circ}\right\|_{2}=0\right) \rightarrow 1
$$

where $\hat{\boldsymbol{\beta}}_{1}^{\circ}$ is the fixed point of $f\left(\boldsymbol{\beta}_{1}\right)$ defined in Lemma S4. Note that $g(\boldsymbol{\beta})$ is a solution to

$$
-\frac{1}{n} D(\boldsymbol{\beta})^{-1} l(\boldsymbol{\theta})+\frac{1}{n} \lambda_{n} \boldsymbol{\theta}=\mathbf{0},
$$

where $D(\boldsymbol{\beta})^{-1}=\operatorname{diag}\left\{\beta_{1}^{2}, \ldots, \beta_{q_{n}}^{2}, \beta_{q_{n}+1}^{2}, \ldots, \beta_{p_{n}}^{2}\right\}$. It is easy to see from (57) that

$$
\lim _{\boldsymbol{\beta}_{2} \rightarrow 0} g_{2}(\boldsymbol{\beta})=\mathbf{0}_{p_{n}-q_{n}}
$$


This, combined with (57), implies that for any $\boldsymbol{\beta}_{1}$

$$
\lim _{\boldsymbol{\beta}_{2} \rightarrow 0} g_{1}(\boldsymbol{\beta})=f\left(\boldsymbol{\beta}_{1}\right) .
$$

Hence, $g(\cdot)$ is continuous and thus uniform continuous on the compact set $\boldsymbol{\beta} \in \mathcal{H}_{n}$. Hence as $k \rightarrow \infty$,

$$
\omega_{k} \equiv \sup _{\left|g_{1}(\boldsymbol{\beta})\right| \in\left[1 / M_{n}, M_{n}\right] q_{n}}\left\|g_{1}\left(\boldsymbol{\beta}_{1}, \hat{\boldsymbol{\beta}}_{2}^{(k)}\right)-f\left(\boldsymbol{\beta}_{1}\right)\right\|_{2} \rightarrow 0,
$$

with probability tending to one. Furthermore,

$$
\left\|\hat{\boldsymbol{\beta}}_{1}^{(k+1)}-\hat{\boldsymbol{\beta}}_{1}^{\circ}\right\|_{2} \leq\left\|g_{1}\left(\hat{\boldsymbol{\beta}}^{(k)}\right)-f\left(\hat{\boldsymbol{\beta}}_{1}^{(k)}\right)\right\|_{2}+\left\|f\left(\hat{\boldsymbol{\beta}}_{1}^{(k)}\right)-\hat{\boldsymbol{\beta}}_{1}^{\circ}\right\|_{2} \leq \omega_{k}+\frac{1}{K_{4}}\left\|\hat{\boldsymbol{\beta}}_{1}^{(k)}-\hat{\boldsymbol{\beta}}_{1}^{\circ}\right\|_{2},
$$

for some $K_{4}>1$, where the last inequality follows from (45) and the definition of $\omega_{k}$. Denote by $a_{k}=\left\|\hat{\boldsymbol{\beta}}_{1}^{(k)}-\hat{\boldsymbol{\beta}}_{1}^{\circ}\right\|_{2}$, we can rewrite 59 as

$$
a_{k+1} \leq \frac{1}{K_{4}} a_{k}+\omega_{k} .
$$

By (58), for any $\epsilon>0$, there exists an $N>0$ such that $\omega_{k}<\epsilon$ for all $k>N$. Therefore for $k>N$,

$$
\begin{aligned}
& a_{k+1} \leq \frac{1}{K_{4}} a_{k}+\omega_{k} \\
& \leq \frac{a_{k-1}}{K_{4}^{2}}+\frac{\omega_{k-1}}{K_{4}}+\omega_{k} \\
& \leq \frac{a_{1}}{K_{4}^{k}}+\frac{\omega_{1}}{K_{4}^{k-1}}+\cdots+\frac{\omega_{N}}{K_{2}^{k-N}}+\left(\frac{\omega_{N+1}}{K_{4}^{k-N-1}}+\cdots+\frac{\omega_{k-1}}{K_{4}}+\omega_{k}\right) \\
& \leq\left(a_{1}+\omega_{1}+\ldots+\omega_{N}\right) \frac{1}{K_{4}^{k-N}}+\frac{1-\left(1 / K_{4}\right)^{k-N}}{1-1 / K_{4}} \epsilon \rightarrow 0, \quad \text { as } k \rightarrow \infty,
\end{aligned}
$$

with probability tending to one. Therefore,

$$
\operatorname{Pr}\left(\lim _{k \rightarrow \infty}\left\|\hat{\boldsymbol{\beta}}_{1}^{(k)}-\hat{\boldsymbol{\beta}}_{1}^{\circ}\right\|_{2}=\mathbf{0}\right)=1
$$


with probability tending to one, or equivalently

$$
\operatorname{Pr}\left(\hat{\boldsymbol{\beta}}_{1}=\hat{\boldsymbol{\beta}}_{1}^{\circ}\right)=1
$$

with probability tending to one. This proves (56) and thus complete the proof of the theorem.

\section{A.4 Statement and proof of the grouping property}

An appealing property of $\ell_{2}$-penalized regression, which does not hold for $\ell_{0}$-penalized regression, is its tendency to shrink correlated covariates toward each other. As an $\ell_{2^{-}}$ based procedure, the BAR method also retains this grouping property for highly-correlated covariates while retaining the sparsity property of $\ell_{0}$.

Theorem 3 (Grouping property) Assume that $\mathbf{Z}=\left(\mathbf{z}_{i}^{\prime}, \ldots \mathbf{z}_{n}^{\prime}\right)$ is standardized. That $i s$, for all $j=1, \ldots, p, \sum_{i=1}^{n} z_{i j}=0, \mathbf{z}_{[, j]}^{\prime} \mathbf{z}_{[, j]}=n-1$, where $\mathbf{z}_{[, j]}$ is the $j$ th column of $\mathbf{Z}$. Suppose the regularity conditions (C1) - (C6) hold and let $\hat{\boldsymbol{\beta}}$ be the BAR estimator. Then for any $\hat{\beta}_{i} \neq 0$ and $\hat{\beta}_{j} \neq 0$,

$$
\left|\hat{\beta}_{i}^{-1}-\hat{\beta}_{j}^{-1}\right| \leq \frac{1}{\lambda_{n}} \sqrt{2\left\{(n-1)\left(1-r_{i j}\right)\right\}} \sqrt{n\left(1+e_{n}\right)^{2}}
$$

with probability tending to one, where $e_{n}=\sum_{i=1}^{n} I\left(\delta_{i}=1\right)$, and $r_{i j}=\frac{1}{n-1} \mathbf{z}_{[, i]}^{\prime} \mathbf{z}_{[, j]}$ is the sample correlation of $\mathbf{z}_{[, i]}$ and $\mathbf{z}_{[, j]}$.

We can see that as $r_{i j} \rightarrow 1$, the absolute difference between $\hat{\beta}_{i}$ and $\hat{\beta}_{j}$ approaches 0 implying that the estimated coefficients of two highly correlated variables will be similar in magnitude. 
Proof: Under Conditions (C1) - (C6), by Theorem 2 we have that $\hat{\boldsymbol{\beta}}=\lim _{k \rightarrow \infty} \hat{\boldsymbol{\beta}}^{(k)}$, where

$$
\hat{\boldsymbol{\beta}}^{(k+1)}=g\left(\hat{\boldsymbol{\beta}}^{(k)}\right)=\arg \min _{\boldsymbol{\beta}}\left\{-2 l_{n}(\boldsymbol{\beta})+\lambda_{n} \sum_{j=1}^{p_{n}} \frac{I\left(\beta_{j} \neq 0\right) \beta_{j}^{2}}{\left(\hat{\beta}_{j}^{(k)}\right)^{2}}\right\} .
$$

Note that

$$
D\left(\hat{\boldsymbol{\beta}}^{(k)}\right)^{-1} i_{n}\left(\hat{\boldsymbol{\beta}}^{(k+1)}\right)=\lambda_{n} \hat{\boldsymbol{\beta}}^{(k+1)} .
$$

Therefore for any $l=i, j$ where $\hat{\beta}_{i} \neq 0, \hat{\beta}_{j} \neq 0$,

$$
\hat{\beta}_{l}^{(k+1)}=\frac{\left(\hat{\beta}_{l}^{(k)}\right)^{2}}{\lambda_{n}} i_{n l}\left(\hat{\boldsymbol{\beta}}^{(k+1)}\right) .
$$

Letting $k \rightarrow \infty, 62$, we have

$$
\hat{\beta}_{l}^{-1}=\frac{1}{\lambda_{n}} i_{n l}(\hat{\boldsymbol{\beta}}) .
$$

Letting $\boldsymbol{\eta}=Z \boldsymbol{\beta}$ we can rewrite the score function

$$
\zeta\left(\eta_{i}\right)=\frac{\partial}{\partial \eta_{i}} l_{n}(\boldsymbol{\eta})=\int_{0}^{\tau} \hat{w}_{i}(s) d N_{i}(s)+\int_{0}^{\tau} \frac{\hat{w}_{i}^{2}(s) Y_{i}(s) \exp \left(\hat{\eta}_{i}\right)}{\sum_{j=1}^{n} \hat{w}_{j}(s) Y_{j}(s) \exp \left(\hat{\eta}_{j}\right)} d \bar{N}(s) \quad i=1, \ldots, n .
$$

Recall that $\hat{w}_{i}(s) Y_{i}(s) \in[0,1]$ for all $i=1, \ldots n$. Then

$$
\left|\zeta\left(\hat{\eta}_{i}\right)\right| \leq\left|N_{i}(1)\right|+\left|\int_{0}^{\tau} \frac{\hat{w}_{i}^{2}(s) Y_{i}(s) \exp \left(\hat{\eta}_{i}\right)}{\sum_{j=1}^{n} \hat{w}_{j}(s) Y_{j}(s) \exp \left(\hat{\eta}_{j}\right)} d \bar{N}(s)\right| \leq 1+e_{n} \quad i=1, \ldots, n,
$$

where $e_{n}=\sum_{i=1}^{n} I\left(\epsilon_{i}=1\right)$. Hence

$$
\|\zeta(\hat{\boldsymbol{\eta}})\|_{2} \leq\left\|\mathbf{1}+e_{n} \mathbf{1}\right\|_{2}=\sqrt{n\left(1+e_{n}\right)^{2}}
$$

Let $\mathbf{z}_{[, i]}$ denote the $i^{\text {th }}$ column of $Z$. Since $Z$ is assumed to be standardized, $\mathbf{z}_{[, i]}^{\prime} \mathbf{z}_{[, i]}=n-1$ and $\mathbf{z}_{[, i]}^{\prime} \mathbf{z}_{[, j]}=(n-1) r_{i j}$, for all $i \neq j$ and where $r_{i j}$ is the sample correlation between $\mathbf{z}_{[, i]}$ and $\mathbf{z}_{[, j]}$. Since

$$
\hat{\beta}_{i}^{-1}=\frac{1}{\lambda_{n}} \mathbf{z}_{[, i]}^{\prime} \zeta(\hat{\boldsymbol{\eta}}) \quad \text { and } \quad \hat{\beta}_{j}^{-1}=\frac{1}{\lambda_{n}} \mathbf{z}_{[, j]}^{\prime} \zeta(\hat{\boldsymbol{\eta}})
$$


we have

$$
\begin{aligned}
\left|\hat{\beta}_{i}^{-1}-\hat{\beta}_{j}^{-1}\right| & =\left|\frac{1}{\lambda_{n}} \mathbf{z}_{[, i]}^{\prime} \zeta(\hat{\boldsymbol{\eta}})-\frac{1}{\lambda_{n}} \mathbf{z}_{[, j]}^{\prime} \zeta(\hat{\boldsymbol{\eta}})\right| \\
& =\left|\frac{1}{\lambda_{n}}\left(\mathbf{z}_{[, i]}-\mathbf{z}_{[, j]}\right)^{\prime} \zeta(\hat{\boldsymbol{\eta}})\right| \\
& \leq \frac{1}{\lambda_{n}}\left\|\left(\mathbf{z}_{[, i]}-\mathbf{z}_{[, j]}\right)\right\|\|\zeta(\hat{\boldsymbol{\eta}})\| \\
& \leq \frac{1}{\lambda_{n}} \sqrt{2\left\{(n-1)-(n-1) r_{i j}\right\}} \sqrt{n\left(1+e_{n}\right)^{2}}
\end{aligned}
$$

for any $\hat{\beta}_{i} \neq 0$ and $\hat{\beta}_{j} \neq 0$. 


\section{A.5 Proof of Theorem 1}

Because $\hat{\boldsymbol{\beta}}$ is a fixed point of $g(\cdot)$ or $\hat{\boldsymbol{\beta}}=g(\hat{\boldsymbol{\beta}})$, we have for $j=1, \ldots, p$

$$
\left\{\tilde{\mathbf{X}}^{\prime} \tilde{\mathbf{X}}+\lambda_{n} D(\hat{\boldsymbol{\beta}})\right\}\left(\left[\begin{array}{c}
\hat{\beta}_{1} \\
\vdots \\
0 \\
\vdots \\
\hat{\beta}_{p}
\end{array}\right]+\left[\begin{array}{c}
0 \\
\vdots \\
\hat{\beta}_{j} \\
\vdots \\
0
\end{array}\right]\right)=\tilde{\mathbf{X}}^{\prime} \tilde{\mathbf{y}}
$$

Alternative, one can rewrite 62 as

$$
\left\{D(\hat{\boldsymbol{\beta}})^{-1} \tilde{\mathbf{X}}^{\prime} \tilde{\mathbf{X}}+\lambda_{n} \mathbf{I}_{p}\right\}\left(\left[\begin{array}{c}
\hat{\beta}_{1} \\
\vdots \\
0 \\
\vdots \\
\hat{\beta}_{p}
\end{array}\right]+\left[\begin{array}{c}
0 \\
\vdots \\
\hat{\beta}_{j} \\
\vdots \\
0
\end{array}\right]\right)=D(\hat{\boldsymbol{\beta}})^{-1} \tilde{\mathbf{X}}^{\prime} \tilde{\mathbf{y}}
$$

By extracting the $j$ th element of $(63)$, we have

$$
\tilde{\mathbf{x}}_{j}^{\prime} \sum_{i \neq j} \tilde{\mathbf{x}}_{i} \hat{\beta}_{i}^{3}+\lambda_{n} \cdot 0+\tilde{\mathbf{x}}_{j}^{\prime} \tilde{\mathbf{x}}_{j} \cdot \hat{\beta}_{j}^{3}+\lambda_{n} \hat{\beta}_{j}=\tilde{\mathbf{x}}_{j}^{\prime} \tilde{\mathbf{y}}^{2} \hat{\beta}_{j}^{2},
$$

Letting $b_{j}^{*}=\tilde{\mathbf{x}}_{j}^{\prime}\left(\tilde{\mathbf{y}}-\sum_{i \neq j} \tilde{\mathbf{x}}_{i} \hat{\beta}_{i}\right)$, simple algebra will allow us to rewrite 64 as

$$
\hat{\beta}_{j}\left(\tilde{\mathbf{x}}_{j}^{\prime} \tilde{\mathbf{x}}_{j} \cdot \hat{\beta}_{j}^{2}-b_{j}^{*} \hat{\beta}_{j}+\lambda_{n}\right)=0
$$

which yields

$$
\hat{\beta}_{j}= \begin{cases}0, & \text { if }\left|b_{j}^{*}\right|<2 \sqrt{\tilde{\mathbf{x}}_{j}^{\prime} \tilde{\mathbf{x}}_{j} \lambda_{n}} \\ \frac{b_{j}^{*}+\operatorname{sign}\left(b_{j}^{*}\right) \sqrt{\left(b_{j}^{*}\right)^{2}-4 \tilde{\mathbf{x}}_{j}^{\prime} \tilde{\mathbf{x}}_{j} \lambda_{n}}}{2 \tilde{\mathbf{x}}_{j}^{\prime} \tilde{\mathbf{x}}_{j}}, & \text { otherwise. }\end{cases}
$$

for $j=1, \ldots, p$. 


\section{A.6 Proof of Lemma 1}

Recall that, for the PSH model, $\tilde{w}_{i k}=\hat{G}\left(X_{i}\right) / \hat{G}\left(X_{k} \wedge X_{i}\right)$. Because $R_{i}=\left\{y:\left(X_{y} \geq\right.\right.$ $\left.\left.X_{i}\right) \cup\left(X_{y} \leq X_{i} \cap \epsilon_{y}=2\right)\right\}, k \in R_{i}$ implies that either $k \in\left\{y:\left(X_{y} \geq X_{i}\right)\right\}$ or $k \in$ $\left\{y:\left(X_{y} \leq X_{i} \cap \epsilon_{y}=2\right)\right\}$. If $k \in\left\{y:\left(X_{y} \geq X_{i}\right)\right\}$, then $\tilde{w}_{i k}=\hat{G}\left(X_{i}\right) / \hat{G}\left(X_{i}\right)=1$. If $k \in\left\{y:\left(X_{y} \leq X_{i} \cap \epsilon_{y}=2\right)\right\}$, then $\tilde{w}_{i k}=\hat{G}\left(X_{i}\right) / \hat{G}\left(X_{k}\right)$. Therefore

$$
\begin{aligned}
\sum_{k \in R_{i}} \tilde{w}_{i k} \exp \left(\eta_{k}\right) & =\sum_{k \in R_{i}(1)} \tilde{w}_{i k} \exp \left(\eta_{k}\right)+\sum_{k \in R_{i}(2)} \tilde{w}_{i k} \exp \left(\eta_{y}\right) \\
& =\sum_{k \in R_{i}(1)} \exp \left(\eta_{k}\right)+\hat{G}\left(X_{i}\right) \sum_{k \in R_{i}(2)} \exp \left(\eta_{k}\right) / \hat{G}\left(X_{k}\right)
\end{aligned}
$$

where $R_{i}(1)=\left\{y:\left(X_{y} \geq X_{i}\right)\right\}$ and $R_{i}(2)=\left\{y:\left(X_{y}<X_{i} \cap \epsilon_{y}=2\right)\right\}$. 


\section{B Supplementary material for Section 2.3}

\section{B.1 BAR regression via cyclic coordinate descent}

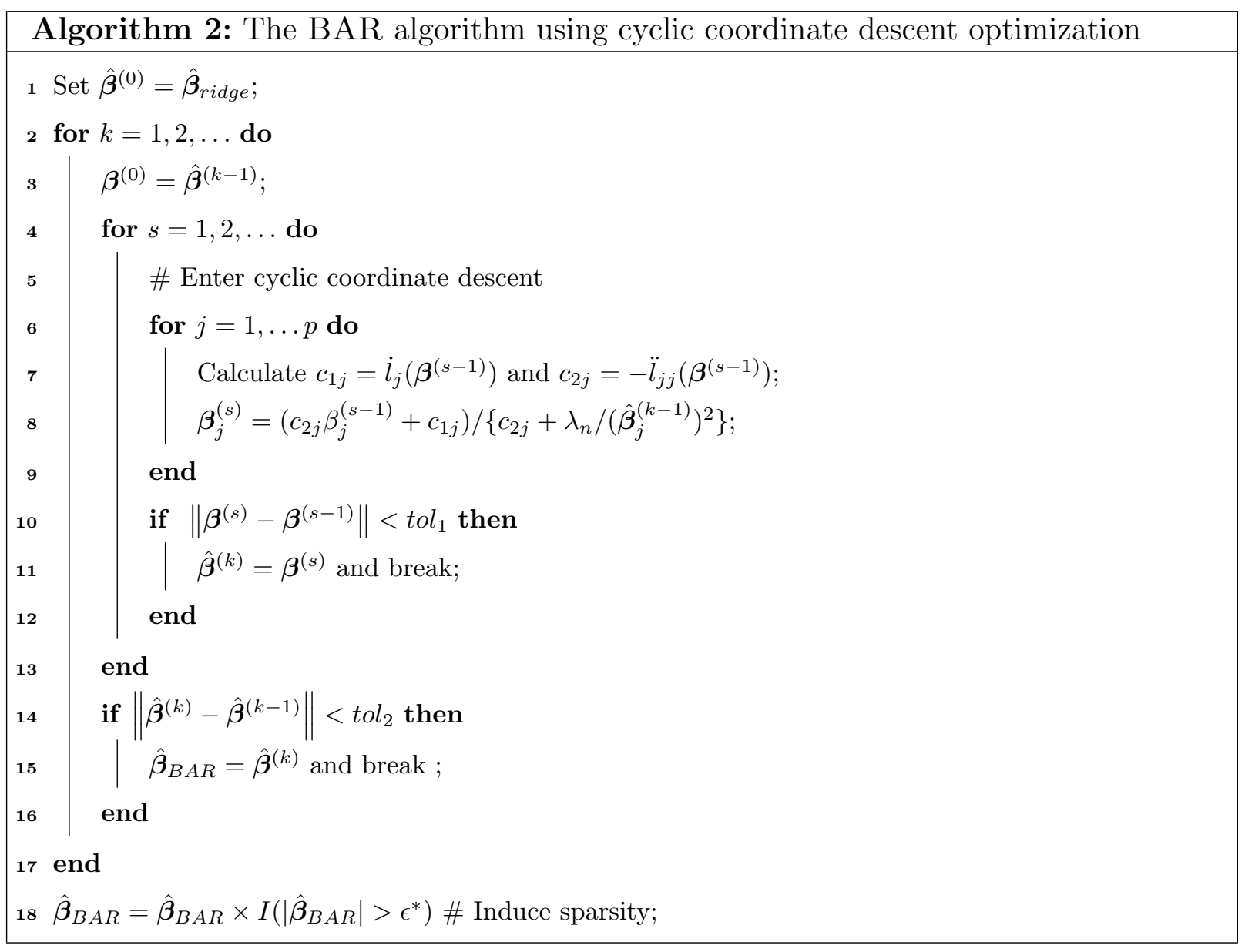

\section{B.2 Computational behavior of cycBAR}

We illustrate under a simple scenario with $p_{n}=2$ that the CYCBAR algorithm converges to the fixed point of $g\left(\beta_{1}, \beta_{2}\right)$ along the graphs of $\beta_{1}=g_{1}\left(\beta_{2}\right)$ and $\beta_{2}=g_{2}\left(\beta_{1}\right)$, with each 
coordinate-wise update moving monotonically a step closer to the fixed point.
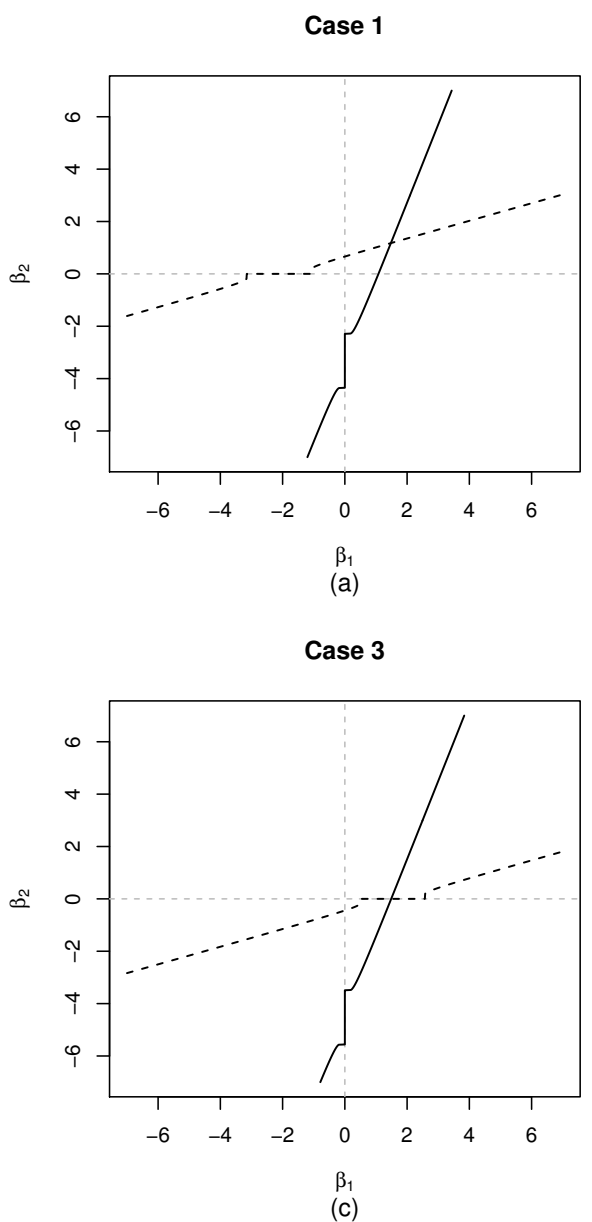

Case 2

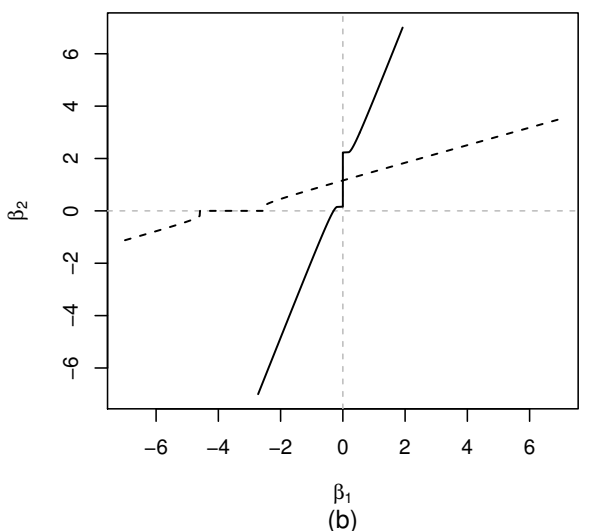

Case 4

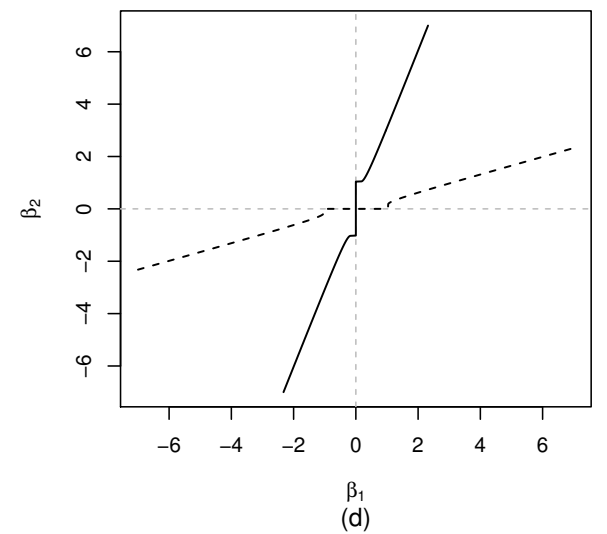

Figure 2: Graphs of $\beta_{1}=g_{1}\left(\beta_{2}\right)$ (solid line) and $\beta_{2}=g_{2}\left(\beta_{1}\right)$ (dotted line) under selected scenarios, which by Theorem 1 , intersect at the fixed-point of $g\left(\beta_{1}, \beta_{2}\right)$. 


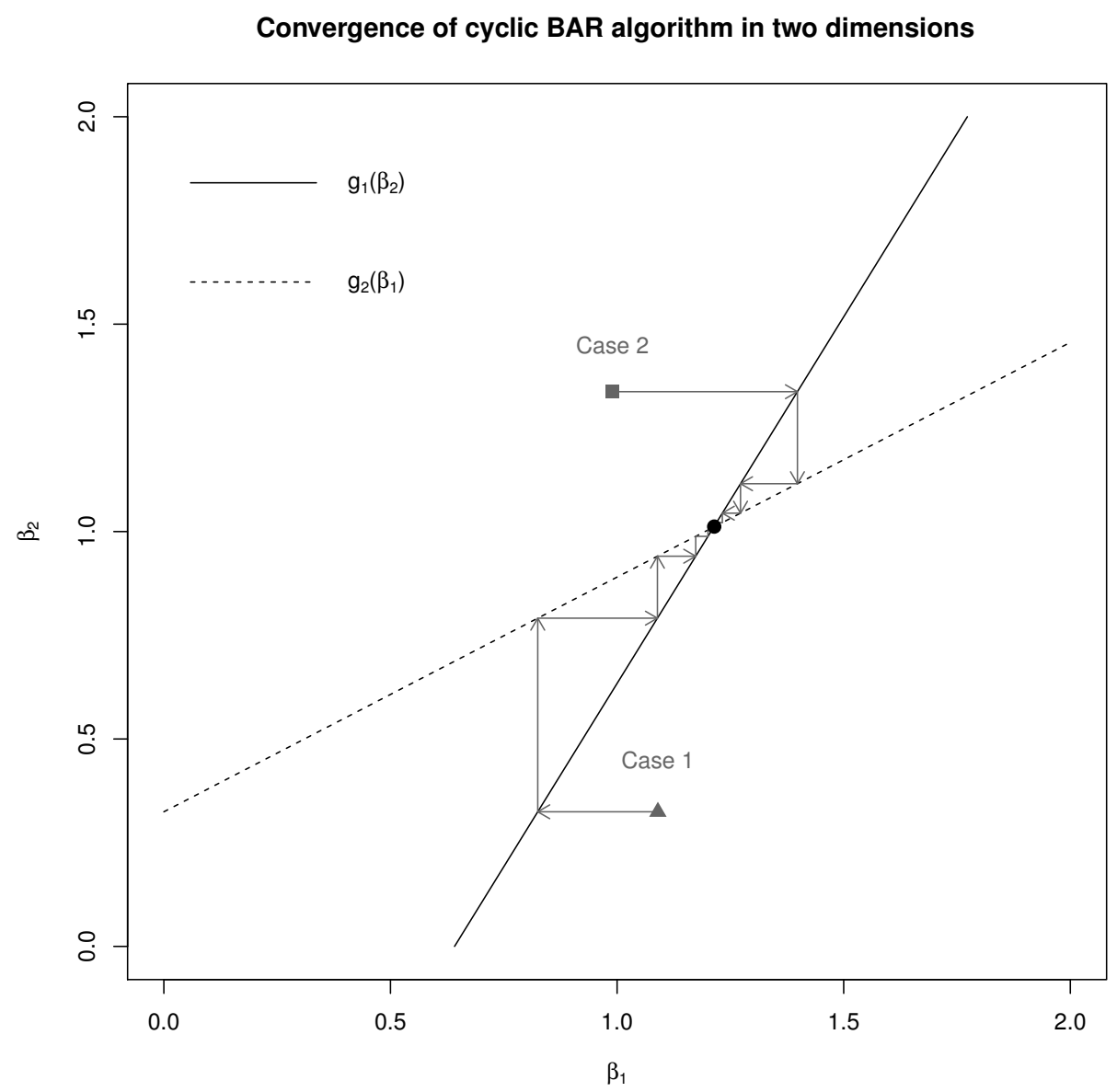

Figure 3: An illustration of the CYCBAR algorithm in a zoomed in picture of Figure S1(a). The BAR estimator is the fixed point of $g\left(\beta_{1}, \beta_{2}\right)$, which, by Theorem 1 , is the intersection of $\beta_{1}=g_{1}\left(\beta_{2}\right)$ and $\beta_{2}=g_{2}\left(\beta_{1}\right)$. 


\section{Supplementary material for Section 3.2}

The operating characteristics of BAR with different tuning parameter selection strategies along with LASSO, adaptive LASSO (ALASSO), SCAD and MCP are assessed by the following measures. As a gold standard, we also fit the oracle model (ORACLE) as if the true model was known a priori. Estimation bias is summarized through the mean squared bias (MSB), $E\left\{\sum_{i=1}^{p}\left(\hat{\boldsymbol{\beta}}_{i}-\boldsymbol{\beta}_{0 i}\right)^{2}\right\}$. Variable selection performance is measured by a number of indices: the mean number of false positives (FP), the mean number of false negatives (FN); and average similarity measure (SM) for support recovery where $S M=\left\|\hat{\mathcal{S}}_{1} \cap \mathcal{S}_{1}\right\|_{0} / \sqrt{\left\|\hat{\mathcal{S}}_{1}\right\|_{0} \cdot\left\|\mathcal{S}_{1}\right\|_{0}}$ and $\mathcal{S}_{1}$ and $\hat{\mathcal{S}}_{1}$ are the set of indices for the non-zero components of $\boldsymbol{\beta}_{1}$ and $\hat{\boldsymbol{\beta}}_{1}$, respectively (Zhang and Cheng, 2017). The similarity measure can be viewed as a continuous measure for true model recovery: it is close to 1 when the estimated model is similar to the true model, and close to 0 when the estimated model is highly dissimilar to the true model.

For BAR, we investigate two tuning parameter selection approaches: 1) $\xi_{n}$ and $\lambda_{n}$ are selected via a two-dimensional grid search to minimize the $\operatorname{BIC}$ criterion $\left(\operatorname{BAR}\left(\xi_{n}, \lambda_{n}\right)\right)$; and 2) $\lambda_{n}$ is selected via grid search to minimize the BIC criterion; and $\xi_{n}=\log \left(p_{n}\right)$ $\left(\operatorname{BAR}\left(\lambda_{n}\right)\right)$. The grids for $\xi_{n}$ and $\lambda_{n}$ were chosen from a log-spaced interval of 25 values between $\left[0.001,3 \log \left(p_{n}\right)\right]$. Unless otherwise noted, we implement BAR using both the CYCBAR and forward-backward scan. The tuning parameter for LASSO, ALASSO, SCAD, and MCP is selected by minimizing the BIC-score through a data-driven grid search of 25 possible values for $\lambda_{n}$. We only consider the $p_{n}<n$ scenario and thus use the maximum pseudo likelihood estimator as the initial estimator for ALASSO.

Tables S1-S3 display the estimation and selection performances of BAR with LASSO, ALASSO, SCAD, and MCP across several simulation scenarios. The selection and estimation performances between optimizing over both $\xi_{n}$ and $\lambda_{n}\left(\operatorname{BAR}\left(\xi_{n}, \lambda_{n}\right)\right)$ and over only 
$\lambda_{n}\left(\operatorname{BAR}\left(\lambda_{n}\right)\right)$ are similar, suggesting that the BAR estimator is insensitive over the choice of $\xi_{n}$. This is further corroborated by Figures S3-S5 where the solution path of the BAR estimator with various choices of are stable over a large interval of $\xi_{n}$. We also observe that BAR and MCP are generally top performers in every scenario and that, as expected, LASSO tends to select more noise variables. 
Table 2: Additional simulation results for model comparison. Based on 100 replications with $\rho=0.5, \boldsymbol{\beta}_{1}=\left(\boldsymbol{\beta}^{*}, \mathbf{0}_{p_{n}-10}\right)$ where $\boldsymbol{\beta}^{*}=(0.40,0.45,0,0.50,0,0.60,0.75,0,0,0.80)$, censoring rate $\approx 33 \%$ and type 1 event rate $\approx 41 \%$.

\begin{tabular}{r|cccc|cccc}
\hline \hline & \multicolumn{4}{|c}{$n=300 ; p=100$} & \multicolumn{4}{c}{$n=700 ; p=100$} \\
\hline Method & MSB & FN & FP & SM & MSB & FN & FP & SM \\
\hline ORACLE & 0.09 & 0.00 & 0.00 & 1.00 & 0.04 & 0.00 & 0.00 & 1.00 \\
\hline $\operatorname{BAR}\left(\xi_{n}, \lambda_{n}\right)$ & 0.31 & 0.40 & 1.87 & 0.85 & 0.06 & 0.01 & 0.89 & 0.94 \\
$\mathrm{BAR}\left(\lambda_{n}\right)$ & 0.32 & 0.49 & 1.70 & 0.85 & 0.06 & 0.01 & 0.86 & 0.94 \\
LASSO & 0.44 & 0.10 & 2.82 & 0.83 & 0.21 & 0.00 & 2.49 & 0.85 \\
ALASSO & 0.39 & 0.75 & 2.00 & 0.81 & 0.09 & 0.00 & 0.73 & 0.95 \\
SCAD & 0.43 & 0.33 & 2.73 & 0.82 & 0.12 & 0.02 & 1.39 & 0.91 \\
MCP & 0.37 & 0.56 & 1.89 & 0.84 & 0.08 & 0.08 & 0.65 & 0.95 \\
\hline
\end{tabular}


Table 3: Additional simulation results for model comparison. Based on 100 replications with $\rho=0.5, \boldsymbol{\beta}_{1}=\left(\boldsymbol{\beta}^{*}, \mathbf{0}_{p_{n}-10}\right)$ where $\boldsymbol{\beta}^{*}=(0.40,0.45,0,0.50,0,0.60,0.75,0,0,0.80)$, censoring rate $\approx 33 \%$ and type 1 event rate $\approx 32 \%(\pi=0.4)$ and $\approx 43 \%(\pi=0.75)$.

\begin{tabular}{r|cccc|cccc}
\hline \hline & \multicolumn{3}{|c}{$n=700 ; p=100 ; \pi=0.4$} & \multicolumn{3}{c}{$n=700 ; p=100 ; \pi=0.75$} \\
\hline Method & MSB & FN & FP & SM & MSB & FN & FP & SM \\
\hline ORACLE & 0.04 & 0.00 & 0.00 & 1.00 & 0.03 & 0.00 & 0.00 & 1.00 \\
\hline BAR $\left(\xi_{n}, \lambda_{n}\right)$ & 0.08 & 0.03 & 0.88 & 0.94 & 0.04 & 0.00 & 0.65 & 0.96 \\
BAR $\left(\lambda_{n}\right)$ & 0.08 & 0.04 & 0.84 & 0.94 & 0.05 & 0.00 & 0.67 & 0.95 \\
LASSO & 0.23 & 0.00 & 2.63 & 0.85 & 0.18 & 0.00 & 2.51 & 0.86 \\
ALASSO & 0.11 & 0.06 & 0.94 & 0.93 & 0.06 & 0.00 & 0.58 & 0.96 \\
SCAD & 0.15 & 0.08 & 1.44 & 0.90 & 0.09 & 0.00 & 0.96 & 0.93 \\
MCP & 0.11 & 0.13 & 0.73 & 0.94 & 0.06 & 0.02 & 0.35 & 0.97 \\
\hline
\end{tabular}
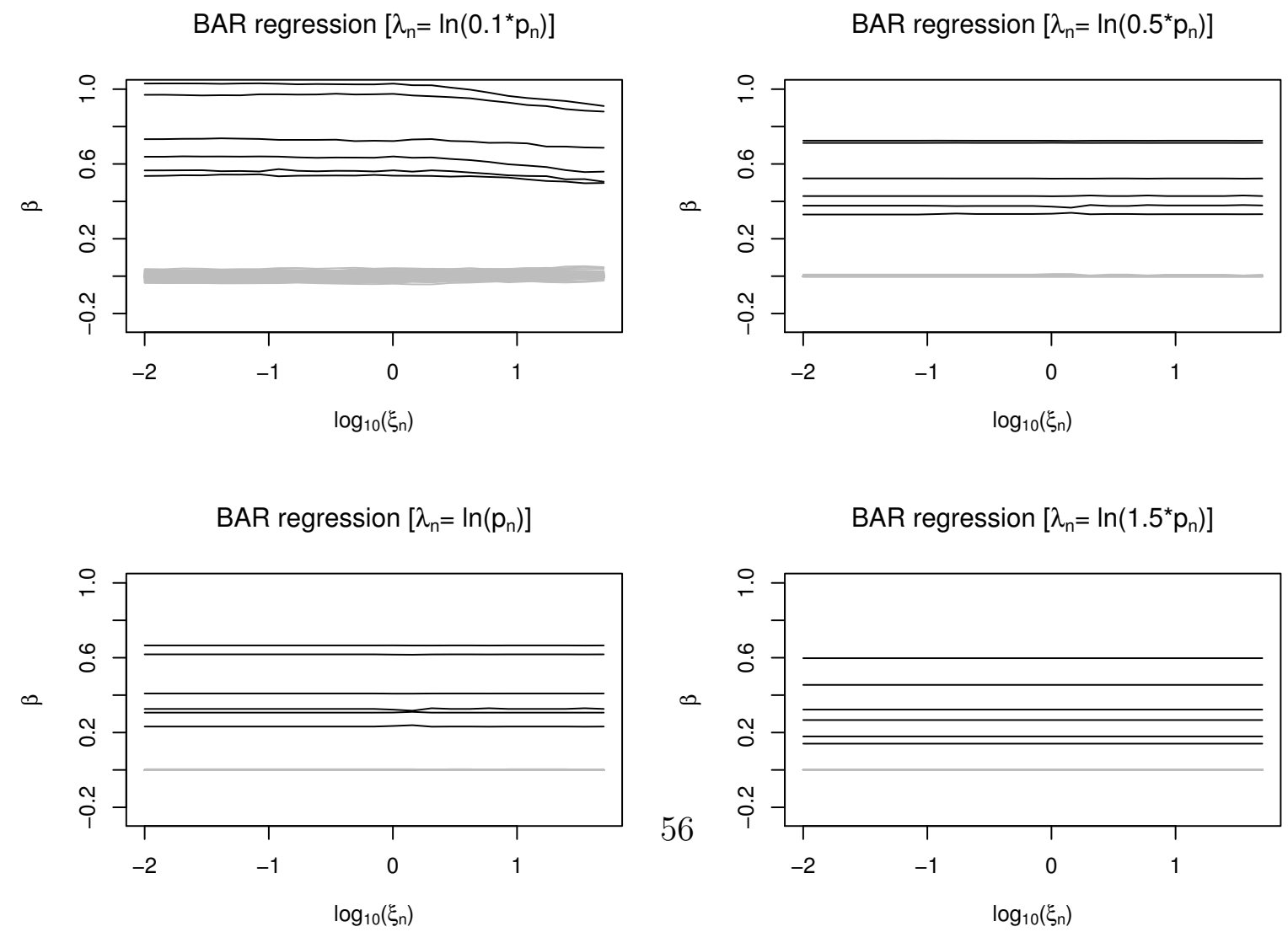

Figure 5: Path plot for BAR regression with varying $\xi_{n}$ and several fixed values of $\lambda_{n}$ where $n=300$ and $p_{n}=100$. The path plots are averaged over 100 simulations. 
Table 4: Additional simulation results for model comparison. Based on 100 replications with $\rho=0.5, \quad \boldsymbol{\beta}_{1}=\left(\boldsymbol{\beta}^{*}, \boldsymbol{\beta}^{*}, \boldsymbol{\beta}^{*}, \mathbf{0}_{p_{n}-30}\right)$ where $\boldsymbol{\beta}^{*}=$ $(0.40,0.45,0,0.50,0,0.60,0.75,0,0,0.80)$, censoring rate $\approx 33 \%$ and type 1 event rate $\approx 41 \%$.

\begin{tabular}{r|cccc|cccc}
\hline \hline & \multicolumn{4}{|c}{$n=300 ; p=100$} & \multicolumn{4}{c}{$n=700 ; p=100$} \\
\hline Method & MSB & FN & FP & SM & MSB & FN & FP & SM \\
\hline ORACLE & 0.40 & 0.00 & 0.00 & 1.00 & 0.13 & 0.00 & 0.00 & 1.00 \\
\hline $\operatorname{BAR}\left(\xi_{n}, \lambda_{n}\right)$ & 0.84 & 0.24 & 3.60 & 0.91 & 0.16 & 0.01 & 1.38 & 0.96 \\
$\operatorname{BAR}\left(\lambda_{n}\right)$ & 0.79 & 0.36 & 3.27 & 0.91 & 0.16 & 0.01 & 1.33 & 0.97 \\
LASSO & 2.32 & 0.05 & 11.02 & 0.79 & 1.27 & 0.00 & 11.92 & 0.78 \\
ALASSO & 1.21 & 0.57 & 6.35 & 0.85 & 0.32 & 0.00 & 2.40 & 0.94 \\
SCAD & 0.98 & 0.19 & 7.00 & 0.85 & 0.16 & 0.01 & 1.54 & 0.96 \\
MCP & 1.03 & 0.33 & 3.59 & 0.91 & 0.15 & 0.02 & 0.73 & 0.98 \\
\hline
\end{tabular}

\section{Supplementary material for Section 3.3}

Table 5: SCAD and MCP penalizations performed with (scan) and without (no scan) the forward-backward scan. Runtime is calculated as mean seconds over 100 simulations.

\begin{tabular}{rr|rrrrrrrr}
\hline \hline & & $n=600$ & 800 & 1000 & 1200 & 1400 & 1600 & 1800 & 2000 \\
\hline \multirow{2}{*}{ SCAD } & (scan) & 0.16 & 0.17 & 0.20 & 0.23 & 0.28 & 0.30 & 0.31 & 0.33 \\
& (no scan) & 4.82 & 7.77 & 12.29 & 15.11 & 23.65 & 35.26 & 34.96 & 42.91 \\
\hline \multirow{2}{*}{$\mathrm{MCP}$} & (scan) & 0.21 & 0.23 & 0.27 & 0.27 & 0.32 & 0.38 & 0.39 & 0.42 \\
& (no scan) & 6.17 & 9.06 & 14.99 & 15.49 & 24.56 & 34.07 & 32.65 & 46.67 \\
\hline
\end{tabular}


Table 6: Additional simulation results for model comparison. Based on 100 replications with $\rho=0.5, \boldsymbol{\beta}_{1}=\left(\boldsymbol{\beta}^{*}, \mathbf{0}_{p_{n}-10}\right)$ where $\boldsymbol{\beta}^{*}=(0.40,0.45,0,0.50,0,0.60,0.75,0,0,0.80)$, censoring rate $\approx 33 \%$ and type 1 event rate $\approx 41 \%$.

\begin{tabular}{|r|cccc|cccc|}
\hline & \multicolumn{4}{|c}{$n=300 ; p=100$} & \multicolumn{4}{c|}{$n=700 ; p=100$} \\
\hline Method & MSB & FN & FP & SM & MSB & FN & FP & SM \\
\hline ORACLE & 0.09 & 0.00 & 0.00 & 1.00 & 0.04 & 0.00 & 0.00 & 1.00 \\
\hline $\operatorname{BAR}\left(\xi_{n}, \lambda_{n}\right)$ & 0.31 & 0.40 & 1.87 & 0.85 & 0.06 & 0.01 & 0.89 & 0.94 \\
$\operatorname{BAR}\left(\lambda_{n}\right)$ & 0.32 & 0.49 & 1.70 & 0.85 & 0.06 & 0.01 & 0.86 & 0.94 \\
$\mathrm{BAR}_{E B I C}$ & 0.51 & 1.68 & 0.03 & 0.84 & 0.10 & 0.25 & 0.00 & 0.98 \\
LASSO & 0.44 & 0.10 & 2.82 & 0.83 & 0.21 & 0.00 & 2.49 & 0.85 \\
ALASSO & 0.39 & 0.75 & 2.00 & 0.81 & 0.09 & 0.00 & 0.73 & 0.95 \\
SCAD & 0.43 & 0.33 & 2.73 & 0.82 & 0.12 & 0.02 & 1.39 & 0.91 \\
MCP & 0.37 & 0.56 & 1.89 & 0.84 & 0.08 & 0.08 & 0.65 & 0.95 \\
\hline
\end{tabular}

\section{E Supplementary material for Section 4}

Table 7: Additional information about the USRDS subset used in Section 4. Summary of event count (\%) observed for the training $(n=125,000)$ and test $(n=100,000)$ sets for the USRDS subset. (Disc: Discontinued dialysis; Recov: Renal function recovery; RC:

Right censored including loss-to-follow up and end of study time.)

\begin{tabular}{|c|c|c|c|c|c|c|}
\hline Set & Transplant & Death & Disc. & Recov. & $\mathrm{RC}$ & Total \\
\hline Training & $11,943(10 \%$ & $60,175(48 \%$ & $8,160(6 \%)$ & $7,555(6 \%)$ & $37,167(30 \%)$ & $125,000(100 \%)$ \\
\hline Test & $9,642(10 \%)$ & $47,830(48 \%)$ & $6,459(7 \%)$ & $6,057(6 \%)$ & $30,012(29 \%)$ & $100,000(100 \%)$ \\
\hline
\end{tabular}



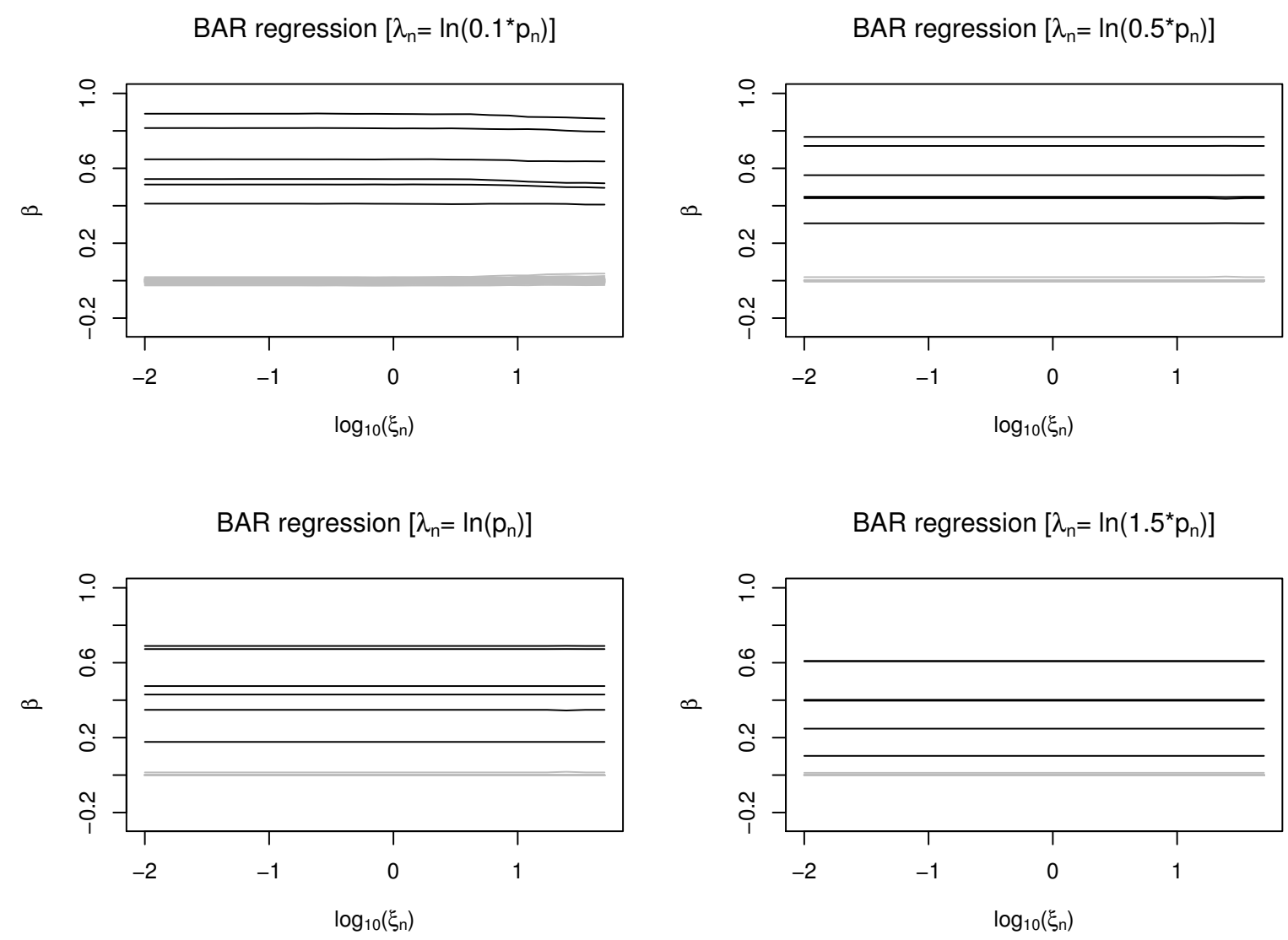

Figure 4: Path plot for BAR regression with varying $\xi_{n}$ and several fixed values of $\lambda_{n}$ where $n=300$ and $p_{n}=40$. The path plots are averaged over 100 simulations. 

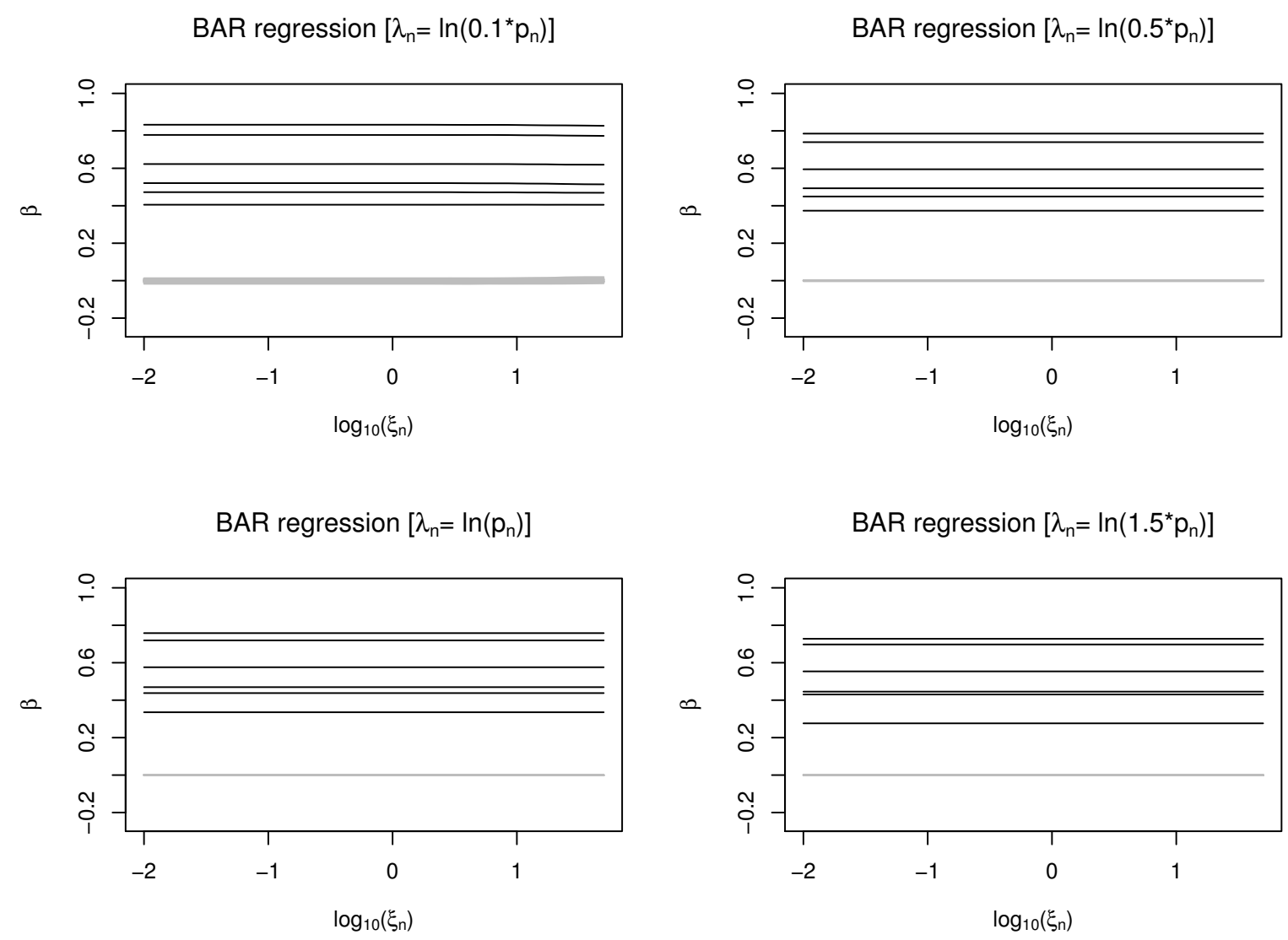

Figure 6: Path plot for BAR regression with varying $\xi_{n}$ and several fixed values of $\lambda_{n}$ where $n=700$ and $p_{n}=40$. The path plots are averaged over 100 simulations. 